\title{
Application of particle methods to static fracture of reinforced concrete structures
}

\author{
T. Rabczuk, T. Belytschko*
}

July 21,2005

Department of Mechanical Engineering, Northwestern University, USA

\begin{abstract}
Particle methods for modeling reinforced concrete are described. The reinforcements are modeled by finite elements and are coupled to the particle method by Lagrange multipliers. The method is applicable to nonlinear problems, problems with moderate to severe cracking and deformable interfaces. Applications to the static response of reinforced concrete structures where the concrete is discretized with particles and the reinforcement with elements are described. The method is also tested for several static problems where no relative displacements between the concrete and the reinforcement are allowed.
\end{abstract}

KEYWORDS: meshfree particle methods; particle-FEM coupling; EFG; fracture; bond; reinforced concrete

\section{INTRODUCTION}

Reinforced concrete structures often undergo extensive cracking before failure. Tracking dense failure patterns by finite element methods is quite difficult. Therefore particle methods are very attractive for this class of problems.

In early approaches, cracks in the concrete were modelled by strain softening in the stress-strain curve, see e.g. Lemaitre [47]. Such continuum models are mesh-dependent if no modifications are made to reflect mesh spacing since a finer discretization leads to a decrease in fracture energy. Other approaches are fictitious crack and smeared crack models, see e.g. Hillerborg et al. [35], Bazant and Oh [9] and Jirasek and Zimmermann [38]. In those models, a crack is assumed to be within an integration cell. Usually the strains are related to a fictitious crack width in the integration cell. In smeared crack models, it has to be distinguished whether a single or several cracks should be modelled within a single integration cell. If larger structures such as shells are of interest, more than one crack can be initiated in an element. The advantage of smeared crack models is that the cracks are initiated through the constitutive model in contrast to discrete crack models; the crack is not considered as a distinct discontinuity.

Non-local approaches as proposed in Bazant et al. [5,6] are another possibility to regularize the constitutive equations. Bazant and Jirasek [8] note that non-local models are also more 'physical' in character. They justify it by micromechanical reasons, e.g. the need to capture size effects observed in experiments. Other approaches are the microplane models, see e.g. Bazant et al. [7]. A good overview about such models can be found in Jirasek [39].

Discrete crack models are an alternative to fictitious crack models. Here, a discontinuity is introduced in the model. One of the possibilities for introducing discrete cracks was studied by Xu and Needleman [60] who separated elements at their boundaries. This approach has the disadvantage that the crack propagation depends on the geometry and the topology of the mesh. Remeshing and refinement can overcome this drawback but they are computationally expensive. The Cornell group of Ingraffea have developed remeshing to a high degree of robustness (see e.g. [50, 20,23]), but three dimensional problems with multiple cracks appear to be very challenging by these methods. Recently, discrete crack methods were developed where the crack can propagate arbitrary in an element without remeshing. Therefore, either the elements or the nodes are enriched with additional degree of freedom. A good overview of embedded elements is given by Jirasek [40]. The latter approach is called the extended finite element method (XFEM) and was developed by Belytschko et al., see e.g. [18, 19].

Meshfree methods are a good alternative to finite elements for crack problems. In addition to the advantage of being more flexible because of the lack of a mesh, they have advantages due to nonlocal interpolation character, higher smoothness and continuity which can be exploited for non-local models for example. Modelling cracks in

${ }^{*}$ T. Belytschko, Department of Mechanical Engineering, Northwestern University, 2145 Sheridan Road, Evanston, IL 60208-311, U.S.A. E-mail: tedbelytschko@northwestern.edu 
meshfree methods was first proposed by Belytschko et al. $[13,14,15]$. The crack was modeled by use of a visibility criterion where the size of the domain of influence was cut when a crack crosses. The concepts of partition-of-unity methods and level sets were incorporated in a meshfree context by Ventura et al. [58].

In this paper, we present further developments of the method for modeling cracking structures presented in Rabczuk and Belytschko [55]. As we show here, the method is capable of treating the nucleation and growth of multiple cracks. Furthermore, the paths of the cracks is not restricted by the numerical model, as in interelement crack models, though the accuracy of the crack path depends on the density of particles. The essential idea underlying this method is to approximate the crack by surfaces (or segments in two dimensions) of discontinuous displacements placed on the particles. The advantage of this approach over level set methods such as Gravouil et al. [32] is that there is no need to track the evolution of the level sets, but this is accomplished at a loss of resolution in the crack surface and crack front. It is also simpler than the triangulation methods employed for crack representation in particle methods by Krysl and Belytschko [46]. In the first versions of the method, the crack surfaces were only placed on the original set of particles. This often resulted in too much roughness of the crack representation so that crack curving and branching was not well reproduced. Here we present a modified method where particles are added in the vicinity of the crack front. This endows the crack with greater continuity and allows better representation of certain phenomena.

Another important issue when modeling reinforced concrete structures is the interaction between the concrete and the reinforcement. If a rigid connection between the concrete and the reinforcement is assumed, the experimental crack pattern can usually be not well reproduced. In reality, the stresses in the reinforcement will increase around the cracked concrete and unloading occurs in the vicinity of the crack which causes cracks at a certain distance from each other. This distance is usually correlated to the bond length which is commonly given as a material parameter determined from experiments. Without a bond model, this effect cannot be captured and cracks will occur over the entire length of the reinforcement in certain applications.

According to Cox and Herrmann [27], bond models can be developed at three different scales, the ribscale, where the geometry of the surface structure of the bar is modelled explicitly, the barscale and the memberscale, where the reinforcement is discretized via a discrete, embedded or smeared model; other novel approaches to model components have also been developed. Typically, in a member scale model, the reinforcement is treated as a onedimensional element, and bond laws have been limited to single-stress models and are not swell suited to reproduce the complicated bond behavior in certain cases. For our applications, models at the barscale are chosen. Cox and Herrmann $[27,28]$ developed a plasticity bond model which can capture both bond failure mechanisms, the pullout failure and the splitting failure. Other bond models were proposed by de Groot et al. [33], Ingraffea et al. [37] or Malvar [48]. We have modified a model originally developed by den Ujil and Bigaj [29] which can also capture both bond failure mechanisms.

Most commonly, bond models are incorporated as interface elements into finite element codes and range from a simple spring to complex interface elements. A good overview of different kind of bond elements is given by Keuser [45]. We will pursue a different approach and couple the finite elements to particles by Lagrange multiplier methods. Elements are used for the reinforcement while particles are used for the concrete. Therefore, we will follow an idea of Hegen [34] who coupled the element-free Galerkin (EFG) method with finite elements via Lagrange multipliers. The method will be extended for nonlinear problems and deformable interfaces, i.e. where relative displacements between the elements and particles are allowed.

The paper is arranged as follows: First, the governing equations will be given. The particle and finite element method and the cracking approach is briefly is reviewed. Then, we derive the coupling approach. It is modified for deformable interfaces. Finally, the coupling approach is tested for a variety of problems.

\section{GOVERNING EQUATIONS}

The governing equation is the equation of equilibrium given in the total Lagrangian description by

$$
\nabla_{0} \cdot \mathbf{P}-\mathbf{b}=\varnothing \quad \forall \mathbf{X} \in \Omega_{0} \backslash \Gamma_{0}^{c}
$$

where $\mathbf{P}$ is the nominal stress tensor (see Belytschko et al. [16] for details), $\mathbf{b}$ are the body forces, $\mathbf{X}$ are the material coordinates, $\nabla_{0}$ is the gradient operator with respect to the material coordinates, $\Gamma_{0}^{c}$ is the crack surface and $\Omega_{0}$ is the domain of the body in the initial configuration. The boundary conditions are

$$
\begin{gathered}
\mathbf{u}(\mathbf{X}, t)=\overline{\mathbf{u}}(\mathbf{X}, t) \quad \text { on } \quad \Gamma_{0}^{u} \\
\mathbf{n}_{0} \cdot \mathbf{P}(\mathbf{X}, t)=\overline{\mathbf{t}}_{0}(\mathbf{X}, t) \quad \text { on } \quad \Gamma_{0}^{t}
\end{gathered}
$$




$$
\mathbf{n}_{0} \cdot \mathbf{P}^{-}=\mathbf{n}_{0} \cdot \mathbf{P}^{+}=\mathbf{t}_{0}^{c} \text { on } \Gamma_{0}^{c}
$$

where $\overline{\mathbf{u}}$ and $\overline{\mathbf{t}}_{0}$ are the prescribed displacements and tractions, respectively, $\mathbf{t}_{0}^{c}$ are the cohesive forces across the crack, $\mathbf{n}_{0}$ is the outward normal to the domain in the initial configuration and $\Gamma_{0}^{u} \bigcup \Gamma_{0}^{t}=\Gamma_{0}, \Gamma_{0}^{u} \cap \Gamma_{0}^{t}=0$. The weak form of the equilibrium equation is

$$
\int_{\Omega_{0}}\left(\nabla_{0} \otimes \delta \mathbf{u}\right)^{T}: \mathbf{P} d \Omega_{0}-\int_{\Omega_{0}} \delta \mathbf{u} \cdot \mathbf{b} d \Omega_{0}-\int_{\Gamma_{0}^{t}} \delta \mathbf{u} \cdot \overline{\mathbf{t}}_{0} d \Gamma_{0}-\int_{\Gamma_{0}^{c}} \mathbf{t}_{0}^{c} \cdot \delta \llbracket \mathbf{u} \rrbracket d \Gamma_{0}=0
$$

where $\mathbf{u}$ and $\delta \mathbf{u}$ are the trial and test functions which should lie in the following spaces

$$
\begin{aligned}
\mathcal{V} & =\left\{\mathbf{u}(\cdot, t) \mid \mathbf{u}(\cdot, t) \in H^{1} \in \Omega_{0} \backslash \Gamma_{0}^{c}, \mathbf{u}(\cdot, t) \text { discontinuous on } \Gamma_{0}^{c} \mathbf{u}(\cdot, t)=\overline{\mathbf{u}}(t) \text { on } \Gamma_{0}^{u}\right\} \\
\mathcal{V}_{0} & =\mathcal{V} \bigcap\left\{\delta \mathbf{u}(\cdot) \mid \delta \mathbf{u}(\cdot)=0 \text { on } \Gamma_{0}^{c}\right\}
\end{aligned}
$$

\section{PARTICLE AND FINITE ELEMENT METHOD}

This section briefly reviews the EFG method and gives the finite element shape functions used in our computations. We will restrict ourselves to $2 \mathrm{D}$ problems.

\subsection{Element-free Galerkin Method}

The EFG-approximation (see $[13,14,15]$ for details) can be written as

$$
u(\mathbf{X}, t)=\mathbf{p}^{T}(\mathbf{X}) \mathbf{a}(\mathbf{X}, t)
$$

where $\mathbf{X}$ are the material coordinates, $t$ is the time and $\mathbf{p}$ are linear basis functions $\mathbf{p}(\mathbf{X})=(1, X, Y)$. Minimizing

$$
J=\sum_{I \in S}\left(\mathbf{p}_{I}^{T}(\mathbf{X}) \mathbf{a}(\mathbf{X}, t)-u_{I}(t)\right)^{2} W\left(\mathbf{X}-\mathbf{X}_{I}, h_{0}\right)
$$

with respect to a leads to the approximation

$$
u(\mathbf{X}, t)=\sum_{I \in S} \Phi_{I}(\mathbf{X}) u_{I}(t)
$$

where $\Phi_{I}(\mathbf{X})$ are the shape functions, $S$ is the set of neighbor particles with $\Phi_{I}(\mathbf{X}) \neq 0$, $u_{I}$ is the value at the particle at the position $\mathbf{X}_{I}, W\left(\mathbf{X}-\mathbf{X}_{I}, h_{0}\right)$ is the weight function and $h$ is the support size of the weight function. After some transformations of equations (7) to (16) we obtain the shape functions

$$
\Phi_{I}=\mathbf{p}^{T}(\mathbf{X}) \cdot \mathbf{A}(\mathbf{X})^{-1} \cdot \mathbf{B}(\mathbf{X})
$$

where

$$
\mathbf{A}(\mathbf{X})=\sum_{I \in S} \mathbf{p}_{I}(\mathbf{X}) \mathbf{p}_{I}^{T}(\mathbf{X}) W\left(\mathbf{X}-\mathbf{X}_{I}, h_{0}\right), \quad \mathbf{B}(\mathbf{X})=\sum_{I \in S} \mathbf{p}_{I}(\mathbf{X}) W\left(\mathbf{X}-\mathbf{X}_{I}, h_{0}\right)
$$

\subsection{FE shape functions}

We use standard bilinear shape functions for four-node finite element:

$$
\Phi_{I}(\xi)=1 / 4\left(1+\xi_{I} \xi\right)\left(1+\eta_{I} \eta\right)
$$

where $\mathbf{X}(\boldsymbol{\xi})$ is a mapping from the parent domain of problem to the physical domain and $\xi_{I}$ and $\eta_{I}$ are the nodal coordinates in the parent domain $\xi \in[-1,1], \eta \in[-1,1]$, given in

$$
\mathbf{x}(\mathbf{X}, t)=\sum_{I=1}^{N} \mathbf{x}_{I}(t) N_{I}(\xi, \eta)
$$

We will also use one dimensional elements such as truss and beam elements. 


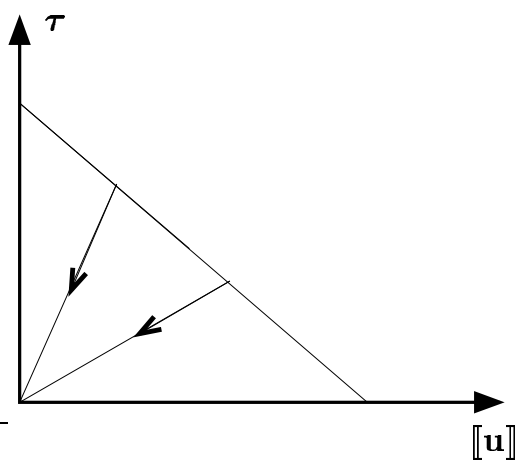

a)

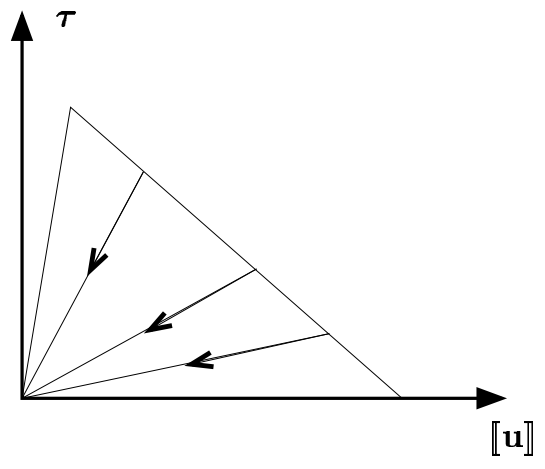

b)

Figure 1: a) Rigid cohesive model, b) non rigid cohesive model

\section{Materials}

\subsection{Constitutive model for the reinforcement}

The reinforcement (steel) is modelled with an elastoplatic constitutive model with isotropic hardening. Details can be found e.g. in Chen [26].

\subsection{Cracking model for concrete}

As mentioned in the introduction, we used a cohesive crack particle method. This approach was proposed by Rabczuk and Belytschko [55] for dynamic problems and will be described here in a static setting.

Starting point is a linear elastic constitutive model (stress-strain curve). We use the Rankine criterion to initiate the crack, i.e. when the stress in the neighborhood of a particle exceeds a given threshold, then a cohesive model is introduced at a particle. Usually, we compare the principal tensile stress to the tensile strength of the material. We used a linear rigid and in some cases a bilinear non-rigid cohesive model as shown in figure 1 . In each case we specify the fracture energy $G_{f}$ and the tensile strength $f_{t}$. A (continuum) scalar damage model was employed in compression. Details can be found in Rabczuk and Eibl [53].

Consider a displacement field which is continuous in the entire domain except at the cracks where a discontinuity occurs in the displacements. To describe this discontinuity, the displacement is decomposed into continuous and discontinuous parts:

$$
\mathbf{u}(\mathbf{X}, t)=\mathbf{u}^{\text {cont }}(\mathbf{X}, t)+\mathbf{u}^{e n r}(\mathbf{X}, t)
$$

where $\mathbf{X}$ are the material coordinates, $t$ is the time, $\mathbf{u}^{\text {cont }}$ denotes the continuous displacement and $\mathbf{u}^{\text {enr }}$ the discontinuous part, which is also called the enrichment.

The crack is modelled by a set of discrete cracks as shown in figure 2. These discrete cracks are restricted to lie on the particles, i.e. each crack line always passes through a particle. Since the crack geometry is described by the set of cracked particles, we do not have to provide a representation for the geometry of the crack.

Let $\mathcal{S}$ be the total set of nodes in the model and $\mathcal{S}_{c}$ the set of cracked nodes. To model the discontinuous part of the displacement, the test and trial functions are enriched with sign functions which are parametrized by $\delta \mathbf{q}_{I}$ and $\mathbf{q}_{I}$, respectively. Only cracked nodes are enriched.

The test and trial functions are

$$
\begin{gathered}
\mathbf{u}^{h}(\mathbf{X}, t)=\sum_{I \in \mathcal{S}} \Phi_{I}(\mathbf{X}) \mathbf{u}_{I}(t)+\sum_{I \in \mathcal{S}_{c}} \Phi_{I}(\mathbf{X}) S\left(f_{I}(\mathbf{X})\right) \mathbf{q}_{I}(t) \\
\delta \mathbf{u}^{h}(\mathbf{X})=\sum_{I \in \mathcal{S}} \Phi_{I}(\mathbf{X}) \delta \mathbf{u}_{I}+\sum_{I \in \mathcal{S}_{c}} \Phi_{I}(\mathbf{X}) S\left(f_{I}(\mathbf{X})\right) \delta \mathbf{q}_{I}
\end{gathered}
$$

where $f_{I}(\mathbf{X})$ is given by

$$
f_{I}(\mathbf{X})=\mathbf{n}_{0} \cdot\left(\mathbf{X}-\mathbf{X}_{I}\right)
$$

where $\mathbf{n}_{0}$ is the normal to the crack and $\mathbf{u}_{I}$ are the particle displacements. 

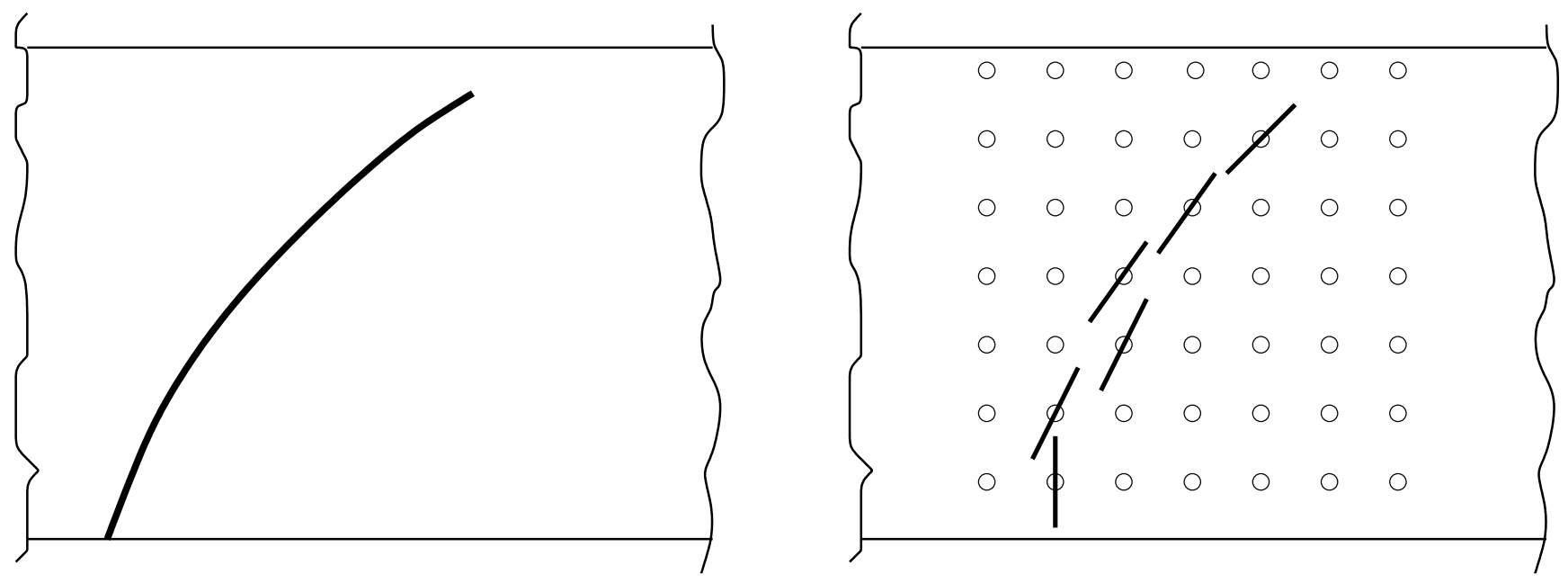

Figure 2: Schematic on the right shows a crack model for the crack on the left

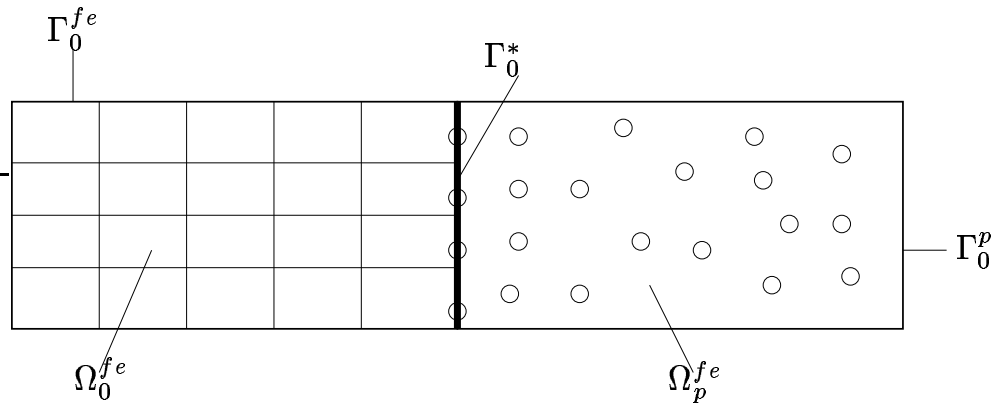

Figure 3: Coupling of particle and finite elements

The sign function $S(\xi)$ is defined as:

$$
S(\xi)=\left\{\begin{array}{cc}
1 & \forall \xi>0 \\
-1 & \forall \xi<0
\end{array}\right.
$$

The nodal displacement vector is extended with $\mathbf{q}$ and the same applies to the vector of internal and external forces as well as to the particle stiffness. More details are given in the next section and in Reference [55].

\section{PARTICLE-FINITE ELEMENT COUPLING}

\subsection{Rigid coupling with Lagrange multipliers}

The equilibrium equations are obtained from a potential. The potential to be minimized is

$$
W=W_{i n t}-W_{e x t}+\lambda^{T} \mathbf{g}
$$

where $W_{i n t}$ is the internal and $W_{\text {ext }}$ is the external energy. The last term on the RHS represents the constraints, i.e. the coupling between the particle and finite element domain. Here, $\boldsymbol{\lambda}$ are the Lagrange multipliers, that can be considered as tractions along the interface $\Gamma_{0}^{*}$, and $\mathbf{g}=\mathbf{u}^{f e}-\mathbf{u}^{p}$ is the gap of the particle and the finite element

domain along the common boundary $\Gamma_{0}^{*}$ as illustrated in figure 3. The Lagrange multipliers are located at the particle positions and are

$$
\mathbf{g}_{h}=\sum_{J=1}^{N} \Phi_{J}^{f e}(\mathbf{X}, t) \mathbf{u}_{J}^{f e}-\sum_{J \in S} \Phi_{J}^{p}(\mathbf{X}, t) \mathbf{u}_{J}^{p}
$$

There are different possibilities to discretize the Lagrange multipliers: 1 . We can place the Lagrange multiplier estimates at the particle position and use finite element shape functions to discretize the Lagrange multiplier field 
$\delta \boldsymbol{\lambda}$ (see figure 4). 2. We can place the Lagrange multiplier estimates at the finite element positions and use the particle shape function to discretize $\delta \boldsymbol{\lambda}$. 3. We can do both. We have chosen the first variant and placed the Lagrange multiplier estimates at the particle positions, see figure 4 . Then, $\delta \boldsymbol{\lambda}^{p}$ is discretized by

$$
\delta \lambda_{h}^{p}(\mathbf{X}, t)=\sum_{J=1}^{N} \Phi_{J}^{f e}(\mathbf{X}, t) \delta \boldsymbol{\Lambda}_{J}(t)
$$

For the interpolation in eq. (21), the position of the Lagrange multipliers in the local element coordinate system has to be known, which is simple in our case since the element is only a "line". The test and trial functions are

$$
\begin{gathered}
\delta \mathbf{u}_{h}(\mathbf{X}, t)=\sum_{J=1}^{N} \Phi_{J}^{f e}(\mathbf{X}, t) \delta \mathbf{u}_{J}^{f e}(t)+\sum_{J \in \mathcal{S}} \Phi_{J}^{p}(\mathbf{X}, t) \delta \mathbf{u}_{J}^{p}(t)+\sum_{I \in \mathcal{S}_{c}} \Phi_{I}^{p}(\mathbf{X}) S\left(f_{I}(\mathbf{X})\right) \delta \mathbf{q}_{I} \\
\mathbf{u}_{h}(\mathbf{X}, t)=\sum_{J=1}^{N} \Phi_{J}^{f e}(\mathbf{X}, t) \mathbf{u}_{J}^{f e}(t)+\sum_{J \in \mathcal{S}} \Phi_{J}^{p}(\mathbf{X}, t) \mathbf{u}_{J}^{p}(t)+\sum_{I \in \mathcal{S}_{c}} \Phi_{I}^{p}(\mathbf{X}) S\left(f_{I}(\mathbf{X})\right) \mathbf{q}_{I}
\end{gathered}
$$

and

$$
\begin{aligned}
& \Phi^{f e}(\mathbf{X}, t)=0 \forall \mathbf{X} \in \Omega_{0}^{p} \\
& \Phi^{p}(\mathbf{X}, t)=0 \forall \mathbf{X} \in \Omega_{0}^{f e}
\end{aligned}
$$

where $\mathcal{S}$ is the set of nodes in the particle model and $\mathcal{S}_{c}$ is the subset of those nodes that are cracked.

Minimizing eq. (19) with respect to $\mathbf{u}$ and $\boldsymbol{\lambda}$ leads to the following equations:

$$
\begin{array}{r}
\frac{\partial W}{\partial \mathbf{u}}=\frac{\partial W_{i n t}}{\partial \mathbf{u}}-\frac{\partial W_{e x t}}{\partial \mathbf{u}}+\boldsymbol{\lambda} \frac{\partial \mathbf{g}}{\partial \mathbf{u}}=\mathbf{f}_{i n t}-\mathbf{f}_{e x t}+\boldsymbol{\lambda} \frac{\partial \mathbf{g}}{\partial \mathbf{u}}=0 \\
\frac{\partial W}{\partial \boldsymbol{\lambda}}=\mathbf{g}=0
\end{array}
$$

The derivatives of $W_{i n t}$ and $W_{e x t}$ with respect to $\mathbf{u}$ are the internal and external forces, respectively:

$$
\begin{gathered}
\mathbf{f}_{i n t}=\int_{\left(\Omega_{0}^{p} \backslash \Gamma_{0}^{c}\right) \cup \Omega_{0}^{f e}}\left(\nabla_{0} \otimes \delta \mathbf{u}\right)^{T}: \mathbf{P} d \Omega_{0} \\
\mathbf{f}_{e x t}=\int_{\left(\Omega_{0}^{p} \backslash \Gamma_{0}^{c}\right) \cup \Omega_{0}^{f e}} \delta \mathbf{u} \cdot \mathbf{b} d \Omega_{0}+\int_{\Gamma_{0}^{p, t} \cup \Gamma_{0}^{f e, t}} \delta \mathbf{u} \cdot \overline{\mathbf{t}}_{0} d \Gamma_{0}+\int_{\Gamma_{0}^{c}} \mathbf{t}_{0}^{c} \cdot \delta \llbracket \mathbf{u} \rrbracket d \Gamma_{0}
\end{gathered}
$$

The additional forces $\boldsymbol{\lambda} \frac{\partial \mathbf{g}}{\partial \mathbf{u}}$ are linear combinations of the Lagrange multipliers. To obtain the discrete system of nonlinear equations we will do a linearization as described e.g. in Belytschko et al. [16]. Therefore, we take a Taylor series expansion of eq. (25) neglecting any higher order terms:

$$
\begin{aligned}
\mathbf{f}_{i n t}-\mathbf{f}_{e x t}+\lambda \frac{\partial \mathbf{g}}{\partial \mathbf{u}}+\frac{\partial \mathbf{f}_{i n t}}{\partial \mathbf{u}} \Delta \mathbf{u}-\frac{\partial \mathbf{f}_{e x t}}{\partial \mathbf{u}} \Delta \mathbf{u}+\frac{\partial \mathbf{g}}{\partial \mathbf{u}} \Delta \boldsymbol{\lambda}+\boldsymbol{\lambda} \frac{\partial^{2} \mathbf{g}}{\partial \mathbf{u} \partial \mathbf{u}} \Delta \mathbf{u}=0 \\
\mathbf{u}+\frac{\partial \mathbf{g}}{\partial \mathbf{u}} \Delta \mathbf{u}=0
\end{aligned}
$$

Substituting the test and trial functions, eqs. (21), (22) and (23) into (28) we finally obtain the following system of equations:

$$
\left[\begin{array}{ccc}
\mathbf{K}^{f e}+\lambda \frac{\partial^{2} \mathbf{g}}{\partial \mathbf{u} \partial \mathbf{u}} & 0 & \mathbf{K}^{f e-f e, T} \\
0 & \mathbf{K}^{p}+\lambda \frac{\partial^{2} \mathbf{g}}{\partial \mathbf{u} \partial \mathbf{u}} & \mathbf{K}^{f e-p, T} \\
\mathbf{K}^{f e-f e} & \mathbf{K}^{f e-p, T} & 0
\end{array}\right] \cdot\left[\begin{array}{c}
\Delta \mathbf{u}_{J}^{f e} \\
\Delta \mathbf{u}_{J}^{p} \\
\Delta \mathbf{\Lambda}
\end{array}\right]=\left[\begin{array}{c}
\mathbf{f}_{e x t}^{f e}-\mathbf{f}_{i n t}^{f e}-\boldsymbol{\lambda}^{T} \mathbf{K}^{f e-f e} \\
\mathbf{f}_{e x t}^{p}-\mathbf{f}_{i n t}^{p}-\boldsymbol{\lambda}^{T} \mathbf{K}^{f e-p} \\
-\mathbf{g}
\end{array}\right]
$$

where $\mathbf{K}^{f e-f e}$ and $\mathbf{K}^{f e-p}$ denotes the derivatives of $\mathbf{g}$ with respect to $\mathbf{u}$ for the finite element $\left(\mathbf{u}^{f e}\right)$ and particle domain $\left(\mathbf{u}^{p}\right)$, respectively and $\mathbf{K}^{f e}$ and $\mathbf{K}^{p}$ are the derivatives of the internal and external forces with respect to 
$\mathbf{u}$. Since neither $\mathbf{b}$ nor the traction boundary conditions $\overline{\mathbf{t}}$ depend on $\mathbf{u}$, the derivatives on $\mathbf{f}_{\text {ext }}$ exist only for the cohesive model. Finally we can give the matrices:

$$
\begin{aligned}
\mathbf{K}^{f e-f e} & =\int_{\Gamma_{0}^{*}} \boldsymbol{\Phi}^{f e, T} \cdot \mathbf{\Phi}^{f e} d \Gamma_{0} \\
\mathbf{K}^{f e-p} & =-\int_{\Gamma_{0}^{*}} \boldsymbol{\Phi}^{f e, T} \cdot \mathbf{\Phi}^{p} d \Gamma_{0} \\
\mathbf{K}^{p} & =\int_{\Omega_{0}^{p} \backslash \Gamma_{0}^{c}}\left(\mathbf{B}^{p, T} \mathbf{C} \mathbf{B}^{p}+\mathbf{B}^{p, T} \mathbf{C ~ B}^{p} S\left(f_{I}(\mathbf{X})\right)\right) d \Omega_{0}+\int_{\Gamma_{0}^{c}} \mathbf{C}^{c o h} \boldsymbol{\Phi}^{p} S\left(f_{I}(\mathbf{X})\right) d \Gamma_{0} \\
\mathbf{K}^{f e} & =\int_{\Omega_{0}^{f e}} \mathbf{B}^{f e, T} \mathbf{C} \mathbf{B}^{f e} d \Omega_{0}
\end{aligned}
$$

and the vectors for internal and external forces

$$
\begin{aligned}
\mathbf{f}_{e x t}^{f e} & =\int_{\Omega_{0}^{f e}} \boldsymbol{\Phi}^{f e, T} \mathbf{b} d \Omega_{0}+\int_{\Gamma_{0}^{f e, t}} \boldsymbol{\Phi}^{f e, T} \overline{\mathbf{t}}_{0} d \Gamma_{0} \\
\mathbf{f}_{e x t}^{p} & =\int_{\Omega_{0}^{p} \backslash \Gamma_{0}^{c}}\left(\boldsymbol{\Phi}^{p, T}+\boldsymbol{\Phi}^{p, T} S\left(f_{I}(\mathbf{X})\right)\right) \mathbf{b} d \Omega_{0}+\int_{\Gamma_{0}^{p, t}}\left(\Phi^{p, T}+\boldsymbol{\Phi}^{p, T} S\left(f_{I}(\mathbf{X})\right)\right) \overline{\mathbf{t}}_{0} d \Gamma_{0} \\
\mathbf{f}_{i n t}^{f e} & =\int_{\Omega_{0}^{f e}} \mathbf{B}^{f e, T} \cdot \mathbf{P} d \Omega_{0} \\
\mathbf{f}_{i n t}^{p} & =\int_{\Omega_{0}^{p} \backslash \Gamma_{0}^{c}}\left(\mathbf{B}^{p, T}+\mathbf{B}^{p, T} S\left(f_{I}(\mathbf{X})\right)\right) \cdot \mathbf{P} d \Omega_{0}
\end{aligned}
$$

where $\mathbf{B}$ is the vector containing the derivatives of the shape functions and $\mathbf{C}^{c o h}$ is the stiffness in the cohesive model. The displacement vector $\Delta \mathbf{u}^{p}=[\Delta \mathbf{u}, \Delta \mathbf{q}]$ is enriched when a new crack segment is added. Since $\mathbf{g}$ is a linear function of the displacement, the second derivatives of $\mathbf{g}$ with respect to $\mathbf{u}$ are zero. Note that we did not incorporate Dirichlet boundary conditions in the particle domain but this procedure is straight forward. Dirichlet boundary conditions such as supports in the FE domain can be imposed directly at the nodes. We use the Newton method to find a stationarity point of a Lagrangian.

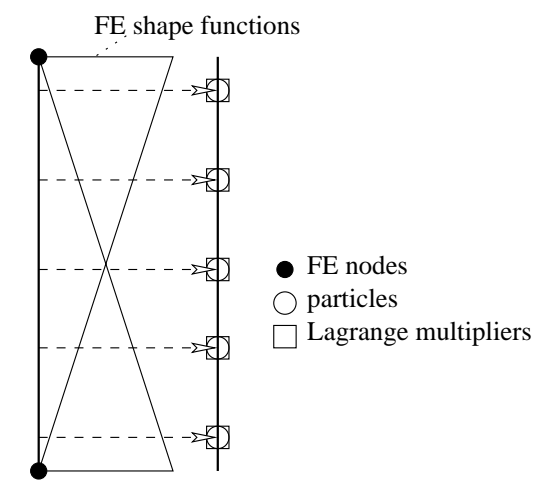

Figure 4: Coupling using the Lagrange multipliers

For linear problems, we obtain the following equations

$$
\left[\begin{array}{ccc}
\mathbf{K}^{f e} & 0 & \mathbf{K}^{f e-f e, T} \\
0 & \mathbf{K}^{p} & \mathbf{K}^{f e-p, T} \\
\mathbf{K}^{f e-f e} & \mathbf{K}^{f e-p, T} & 0
\end{array}\right] \cdot\left[\begin{array}{c}
\mathbf{u}_{J}^{f e} \\
\mathbf{u}_{J}^{p} \\
\boldsymbol{\Lambda}
\end{array}\right]=\left[\begin{array}{c}
\mathbf{f}_{e x t}^{f e} \\
\mathbf{f}_{e x t}^{p} \\
-\mathbf{g}
\end{array}\right]
$$

\subsubsection{Deformable interface coupling}

In this section we will modify the approach of the last section to allow relative displacements along $\Gamma_{0}^{*}$ and call this deformable interface coupling. Therefore, compatibility between the displacement along $\Gamma_{0}^{*}$ is no longer 
required. Instead, traction boundary conditions are applied depending on the relative displacements between the finite element nodes and the particles $\left(\mathbf{u}^{f e}-\mathbf{u}^{p}\right)$ :

$$
\begin{array}{r}
\mathbf{t}^{f e}=\mathbf{k}^{*}\left(\mathbf{u}^{f e}-\mathbf{u}^{p}\right)=\mathbf{k}^{*} \overline{\mathbf{g}} \text { on } \Gamma_{0}^{*} \\
\mathbf{t}^{p}=-\mathbf{k}^{*}\left(\mathbf{u}^{f e}-\mathbf{u}^{p}\right)=-\mathbf{k}^{*} \overline{\mathbf{g}} \text { on } \Gamma_{0}^{*}
\end{array}
$$

where $\mathbf{k}^{*}$ can be considered as spring stiffness between the two domains. The spring stiffness depends on the relative displacement $\overline{\mathbf{g}}=\left(\mathbf{u}^{f e}-\mathbf{u}^{p}\right)$ and some internal variables. Using (33), we have:

$$
W=W_{i n t}-W_{e x t}-W^{*}+\lambda^{T} \mathbf{g}
$$

where $W^{*}$ can be considered as external energy due to forces across the finite element-particle interface and the last term of the right hand side usually vanishes if normal and tangential relative displacements are allowed. The test and the trial functions are the same as in the previous section. Minimizing eq. (34) with respect to $\mathbf{u}$ and $\boldsymbol{\lambda}$ gives

$$
\begin{array}{r}
\frac{\partial W}{\partial \mathbf{u}}=\frac{\partial W_{i n t}}{\partial \mathbf{u}}-\frac{\partial W_{e x t}}{\partial \mathbf{u}}-\frac{\partial W^{*}}{\partial \mathbf{u}}+\boldsymbol{\lambda} \frac{\partial \mathbf{g}}{\partial \mathbf{u}}=\mathbf{f}_{i n t}-\mathbf{f}_{e x t}-\mathbf{f}^{*}+\boldsymbol{\lambda} \frac{\partial \mathbf{g}}{\partial \mathbf{u}}=0 \\
\frac{\partial W}{\partial \boldsymbol{\lambda}}=\mathbf{g}=0
\end{array}
$$

with the forces across the interface boundary

$$
\begin{aligned}
& \left(\mathbf{f}^{*}\right)_{I}^{f e}=\int_{\Gamma_{0}^{*}} \boldsymbol{\Phi}_{I}^{f e, T} \cdot \mathbf{k}^{*} \overline{\mathbf{g}} d \Gamma_{0} \\
& \left(\mathbf{f}^{*}\right)_{I}^{p}=-\int_{\Gamma_{0}^{*}} \boldsymbol{\Phi}_{I}^{p, T} \cdot \mathbf{k}^{*} \overline{\mathbf{g}} d \Gamma_{0}
\end{aligned}
$$

Note, that we can allow relative displacements tangential to $\Gamma_{0}^{*}$ while no relative displacements normal to $\Gamma_{0}^{*}$ are allowed or vice versa. For this general case, the following equations hold:

$$
\begin{aligned}
\mathbf{f}_{I}^{i n t}-\mathbf{f}_{I}^{e x t}-\mathbf{f}_{I}^{*}+\lambda \frac{\partial \mathbf{g}}{\partial \mathbf{u}_{I}} & =0 \\
\mathbf{g} & =0
\end{aligned}
$$

where the indices $I$ indicate either the particle or FE domain. Note, that the Lagrange multipliers vanish if we allow relative displacements normal and tangential along $\Gamma_{0}^{*}$. Taking a Taylor series expansion of eq. (38) we obtain

$$
\begin{aligned}
\mathbf{f}_{I}^{i n t}-\mathbf{f}_{I}^{e x t}-\mathbf{f}_{I}^{*}+\lambda \frac{\partial \mathbf{g}}{\partial \mathbf{u}_{I}}+\frac{\partial \mathbf{f}^{i n t}}{\partial \mathbf{u}_{I}} \Delta \mathbf{u}_{I}-\frac{\partial \mathbf{f}^{e x t}}{\partial \mathbf{u}_{I}} \Delta \mathbf{u}_{I}-\frac{\partial \mathbf{f}^{*}}{\partial \mathbf{u}_{I}} \Delta \mathbf{u}_{I}+\frac{\partial \mathbf{g}}{\partial \mathbf{u}_{I}} \Delta \boldsymbol{\lambda}+\lambda \frac{\partial^{2} \mathbf{g}}{\partial \mathbf{u}_{I} \partial \mathbf{u}_{I}} \Delta \mathbf{u}_{I} & =0 \\
\mathbf{u}_{I}+\frac{\partial \mathbf{g}}{\partial \mathbf{u}_{I}} \Delta \mathbf{u}_{I} & =0
\end{aligned}
$$

The derivatives of $\mathbf{f}^{*}$ with respect to the displacement can be written as

$$
\frac{\partial \mathbf{f}_{I}^{*}}{\partial \mathbf{u}_{I}}=\overline{\mathbf{g}} \frac{\partial \mathbf{k}^{*}}{\partial \mathbf{u}_{I}}+\mathbf{k}^{*} \frac{\partial \overline{\mathbf{g}}}{\partial \mathbf{u}_{I}}
$$

For a constant spring stiffness, the first term on the RHS vanishes and the coupling matrix is

$$
\begin{aligned}
& \left(\mathbf{K}^{*}\right)^{f e-f e}=\int_{\Gamma_{0}^{*}} \boldsymbol{\Phi}^{f e, T} \cdot \mathbf{k}^{*} \cdot \mathbf{\Phi}^{f e} d \Gamma_{0} \\
& \left(\mathbf{K}^{*}\right)^{f e-p}=-\int_{\Gamma_{0}^{*}} \boldsymbol{\Phi}^{f e, T} \cdot \mathbf{k}^{*} \cdot \boldsymbol{\Phi}^{p} d \Gamma_{0}
\end{aligned}
$$

If the spring stiffness depends on the relative displacements, an expression for $\frac{\partial \mathbf{k}^{*}}{\partial \mathbf{u}^{I}}$ has to be given. For constant $\mathbf{k}^{*}$, we obtain

$$
\left[\begin{array}{ccc}
\mathbf{K}^{f e}+\left(\mathbf{K}^{*}\right)^{f e-f e} & 0 & \mathbf{K}^{f e-f e, T} \\
0 & \mathbf{K}^{p}+\left(\mathbf{K}^{*}\right)^{f e-p} & \mathbf{K}^{f e-p, T} \\
\mathbf{K}^{f e-f e} & \mathbf{K}^{f e-p, T} & 0
\end{array}\right] \cdot\left[\begin{array}{c}
\Delta \mathbf{u}_{J}^{f e} \\
\Delta \mathbf{u}_{J}^{p} \\
\Delta \mathbf{\Lambda}
\end{array}\right]=\left[\begin{array}{c}
\mathbf{f}_{e x t}^{f e}-\mathbf{f}_{i n t}^{f e}+\left(\mathbf{f}^{*}\right)^{f e}-\boldsymbol{\lambda}^{T} \mathbf{K}^{f e-f e} \\
\mathbf{f}_{e x t}^{p}-\mathbf{f}_{i n t}^{p}-\left(\mathbf{f}^{*}\right)^{p}-\boldsymbol{\lambda}^{T} \mathbf{K}^{f e-p} \\
-\mathbf{g}
\end{array}\right]
$$


If we allow relative displacements in both directions, tangential and normal to $\Gamma_{0}^{*}$, eq. (43) simplifies to

$$
\left[\begin{array}{cc}
\mathbf{K}^{f e}+\left(\mathbf{K}^{*}\right)^{f e-f e} & 0 \\
0 & \mathbf{K}^{p}+\left(\mathbf{K}^{*}\right)^{f e-p}
\end{array}\right] \cdot\left[\begin{array}{c}
\Delta \mathbf{u}_{J}^{f e} \\
\Delta \mathbf{u}_{J}^{p}
\end{array}\right]=\left[\begin{array}{c}
\mathbf{f}_{e x t}^{f e}-\mathbf{f}_{i n t}^{f e}+\left(\mathbf{f}^{*}\right)^{f e} \\
\mathbf{f}_{e x t}^{p}-\mathbf{f}_{i n t}^{p}-\left(\mathbf{f}^{*}\right)^{p}
\end{array}\right]
$$

Note, if $\left(\mathbf{K}^{*}\right)^{f e-f e}>>\mathbf{K}^{f e}$ and $\left(\mathbf{K}^{*}\right)^{f e-p}>>\mathbf{K}^{p}$ we obtain a rigid coupling using the penalty method.

The discrete equations are evaluated by using Gauss quadrature for both the finite element and particle domain. For the particle method, a background mesh is used where the particle form rectangular background cells; $4 \times 4$ Gauss points were used in each integration cell.

\section{The Bond Mechanism}

The bond behavior depends on the surface of the reinforcement bars. For bars without ribs, adhesion and friction are the principal mechanisms of bonding. For ribbed bars, the bond behavior is concentrated around a very small domain, approximately of the size of the ribs. We are mainly interested in bars with ribs.

Adhesion breaks down relatively early in the bond interface response so that the bond force is transferred by friction and the mechanical interaction of the ribs with the adjacent concrete. The concrete next to the ribs fails either by crushing due to the pressure of the ribs on the concrete or by transverse cracking that initiate at the ribs, see figure 5.

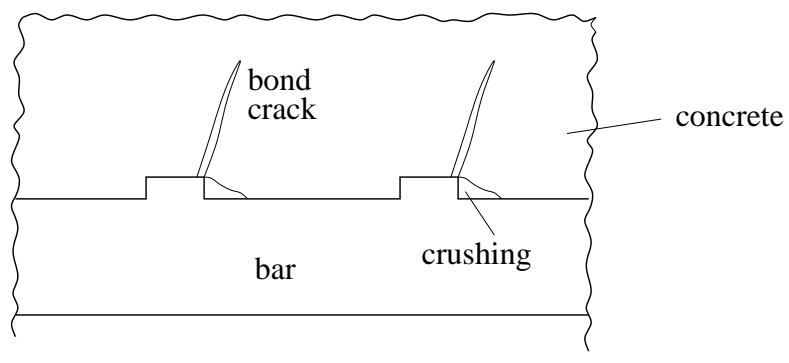

a)

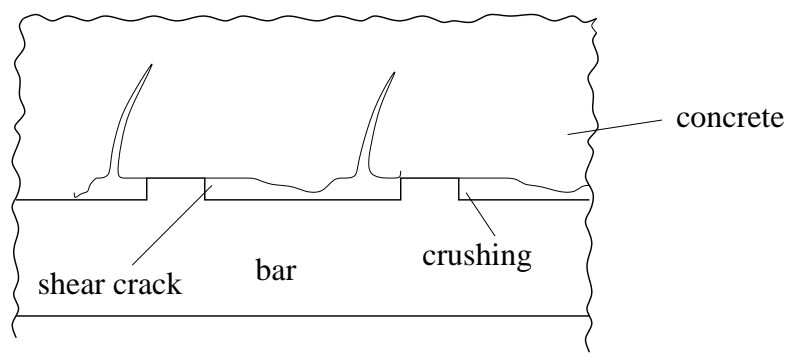

b)

Figure 5: Bond mechanism

Radial splitting forces can occur which are due to the rotation of the inclined struts which produce a larger radial component of the contact forces. Without a sufficient concrete cover throughout, a splitting failure can occur.

\section{The bond model}

The bond failure is idealized into two types of failure: a pullout failure, where the bond strength in the contact zone is exceeded, and a splitting failure which is often caused by an insufficient concrete cover throughout. Direction changes of the reinforcement promote the latter failure mechanism, too. We modified an approach proposed by Den Uijl and Bigaj [29]. This approach is able to model both failure mechanisms, a pullout and a splitting failure. Furthermore, the model is extended to reproduce a splitting tensile failure which is especially important for structures with changes of the reinforcement.

\subsection{Splitting failure}

The forces are transmitted from the bar to the concrete by the ribs of the bar. This causes cone-shaped radial cracks in the concrete. In the bond model, an initiation of three radial cracks as shown in figure 6 is assumed. The most important parameter is the quotient $\xi=c_{e f f} / D$ of the concrete cover $c_{e f f}$ and the diameter $D$ of the rod. If the radial cracks pass through the complete concrete cover throughout, a sudden failure takes place. The bond model is formulated in terms of the radial stress-radial strain relation. This curve can be split into three domains. The first domain describes the nonlinear material behavior caused by the crack propagation, the second domain is the linear softening domain and the third one is characterized by the residual strength. 


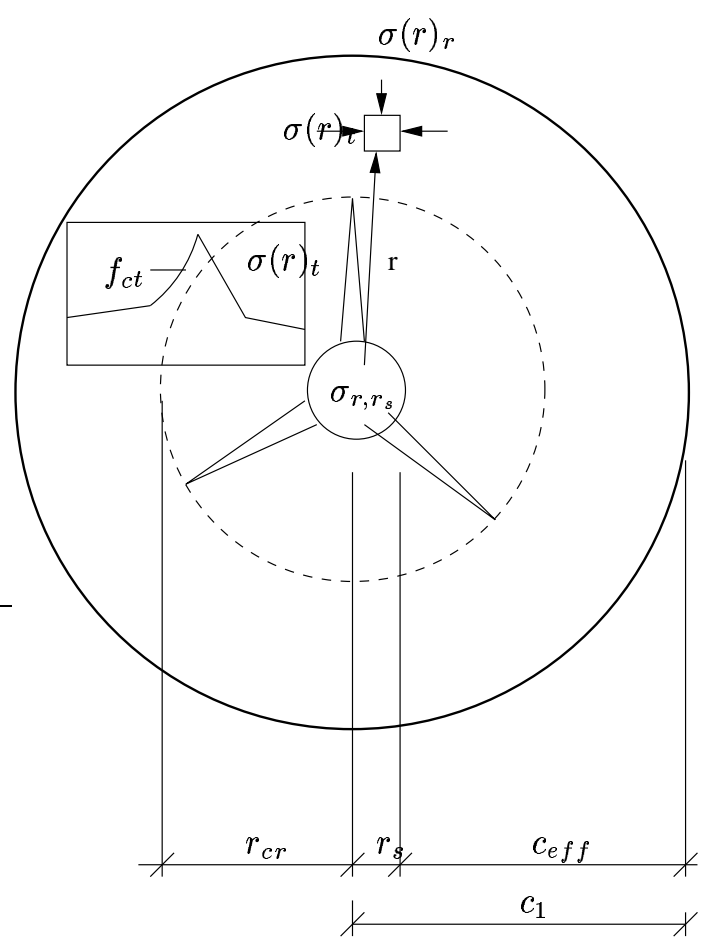

Figure 6: Bond model with three radial cracks in the concrete

Domain $1: 0 \leq \epsilon_{r, r_{s}} \leq \epsilon_{r, r_{s}, \max }$

$$
\sigma_{r, r_{s}}\left(\epsilon_{r, r_{s}}\right)=\sigma_{r, r_{s}, \max } \frac{k \eta-\eta^{2}}{1+(k-2) \eta}
$$

with

$$
k=\frac{E_{r} \epsilon_{r, r_{s}, \max }}{\sigma_{r, r_{s}, \max }} \quad \eta=\frac{\epsilon_{r, r_{s}}}{\epsilon_{r, r_{s}, \max }}
$$

and the maximum radial stress $\sigma_{r, r_{s}, \max }$ and strain $\epsilon_{r, r_{s}, \text { max }}$, respectively, at failure (see Akkermann, 2001)

$$
\sigma_{r, r_{s}, \max }=2 \xi^{0.88} f_{c t} \quad, \quad \epsilon_{r, r_{s}, \max }=4.2 \xi^{1.08} \frac{f_{c t}}{E_{0}}
$$

where $E_{0}$ is the Young's modulus, $f_{c t}$ is the tensile strength of concrete, $\xi=c_{e f f} / D$ where $c_{e f f}$ is the concrete cover throughout and $D$ is the diameter of the rod. The initial stiffness in radial direction is:

$$
E_{r}=E_{0}\left(\frac{\left(c_{e f f}+D / 2\right)^{2}+D^{2} / 4}{\left(c_{e f f}+D / 2\right)^{2}-D^{2} / 4}+\nu\right)^{-1}
$$

Domain $2: \epsilon_{r, r_{s}, \max }<\epsilon_{r, r_{s}} \leq \epsilon_{r, r_{s}, \text { res }}$

$$
\sigma_{r, r_{s}}\left(\epsilon_{r, r_{s}}\right)=\sigma_{r, r_{s}, \max }\left(1-\frac{1-\psi}{\epsilon_{r, r_{s}, r e s}-\epsilon_{r, r_{s}, \max }}\left(\epsilon_{r, r_{s}}-\epsilon_{r, r_{s}, \max }\right)\right), \psi=0.2
$$

with

$$
\epsilon_{r, r_{s}, r e s}=\left(2 \xi+c_{0} / D\right) \frac{f_{c t}}{E_{0}}, \quad c_{0}=0.27 m
$$

Domain $3: \epsilon_{r, r_{s}, r e s}<\epsilon_{r, r_{s}}$

$$
\sigma_{r, r_{s}}\left(\epsilon_{r, r_{s}}\right)=\sigma_{r, r_{s}, \max }=\psi \sigma_{r, r_{s}, r e s}, \quad \psi=0.2
$$

The bond stresses and the slip has to be defined parallel to the reinforcement. The transmission of the forces from the reinforcement into the concrete is considered as shown in figure 7 . The radial strains can be computed from the slip $\delta_{p}$ by

$$
\epsilon_{r, r_{s}}\left(\delta_{p}\right)=\frac{2 \delta_{p}}{D} \tan \vartheta_{b} \text { with } \vartheta_{b}=0.1 f_{c}
$$




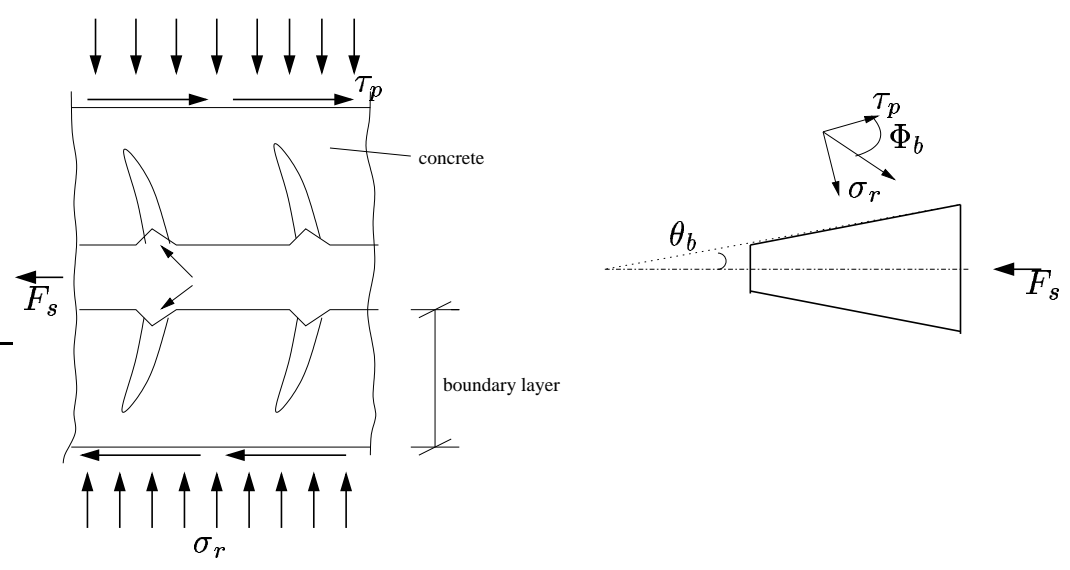

Figure 7: Bond model

where $f_{c}$ is the compressive strength of the concrete. The bond stress is coupled with the radial stress by a fictitious friction

$$
\tau_{p}=\cot \Phi \sigma_{r, r_{s}}
$$

The friction angle depends on the slope of the circumferential cracks and is approximated by $\cot \Phi=1$. Hence, the bond slip relation is completely determined for a splitting failure.

\subsection{Pullout failure}

For a pullout failure, the slip depends on $\vartheta_{b}$, which decreases with increasing damage of the concrete since the shear resistance of concrete will decrease, too. Finally, the radial strain can be formulated as function of the slip $\delta_{p}$ and the steel strains $\epsilon_{s}$

$$
\epsilon_{r, r_{s}}=\frac{f\left(\delta_{p}, \epsilon_{s}\right)}{r_{s}}
$$

The function $f\left(\delta_{p}, \epsilon_{s}\right)$ is divided into four parts. A detailed description can be found in Den Uijl, Bigaj [29].

The bond stresses are computed depending on the relevant failure mechanism. Figure 8a shows the bond slip relations for splitting failure, figure $8 \mathrm{~b}$ for a pullout failure. For the splitting failure, $\tan \vartheta_{b}$ is constant. Hence, the radial strains are linearly dependent on the slip. For the pullout failure, the radial strains are nonlinearly dependent on the slip. If the radial stresses are smaller than the maximum slip stresses $\sigma_{r, r_{s}, \max } \leq \tau_{p, \max }$ with $\tau_{p, \max }=5 f_{c t}$, a splitting failure takes place, otherwise a pullout failure occurs. The radial strains are calculated according to eq. (51) or (53) corresponding to the failure mechanism. Once the radial strains are computed, the radial stresses are obtained, which are equal to the bond stresses.

\subsection{Splitting tensile failure}

Changes in the direction of the reinforcement lead to returning pressing as shown in figure 10. If the tensile strength of the concrete is exceeded and without any further arrangements, the structure will fail suddenly due to spalling of the concrete cover throughout. Figure 9 shows a typical splitting tensile failure.

Though the bond behavior is three-dimensional, it can be modelled in $2 \mathrm{D}$ since the mechanical behavior is described by a stress-deformation relation perpendicular to the reinforcement. It can be shown that the forces which result from the curved reinforcement can be approximated by linear framework elements according to figure 10b:

$$
\int_{-\varphi}^{\varphi} p_{u} 0.5 D_{b r} \cos \varphi d \varphi=2 p_{u} 0.5 D_{b r} \sin \varphi
$$

where $D_{b r}$ is defined in figure 10a. We tried to model the splitting tensile failure empirically based on experimental observation since analytical conclusion which describe the complicated splitting tensile failure properly are difficult to derive.

It can be assumed that the relative displacements between the concrete and the reinforcement perpendicular to the axis are small compared to the deformations of the surrounding concrete until the maximum returning pressing 


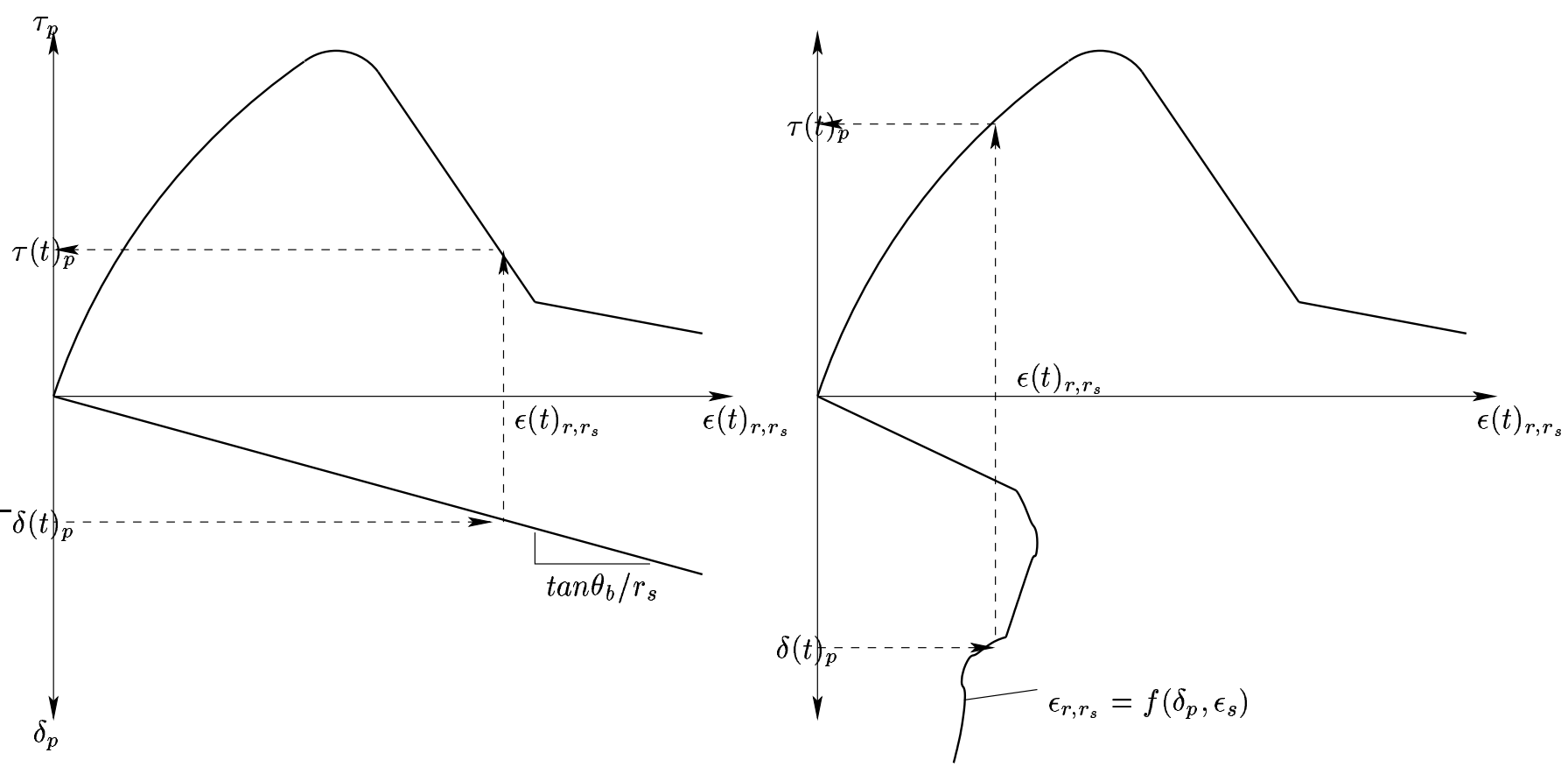

Figure 8: Bond stress- slip relation for a) splitting failure, b) pullout failure

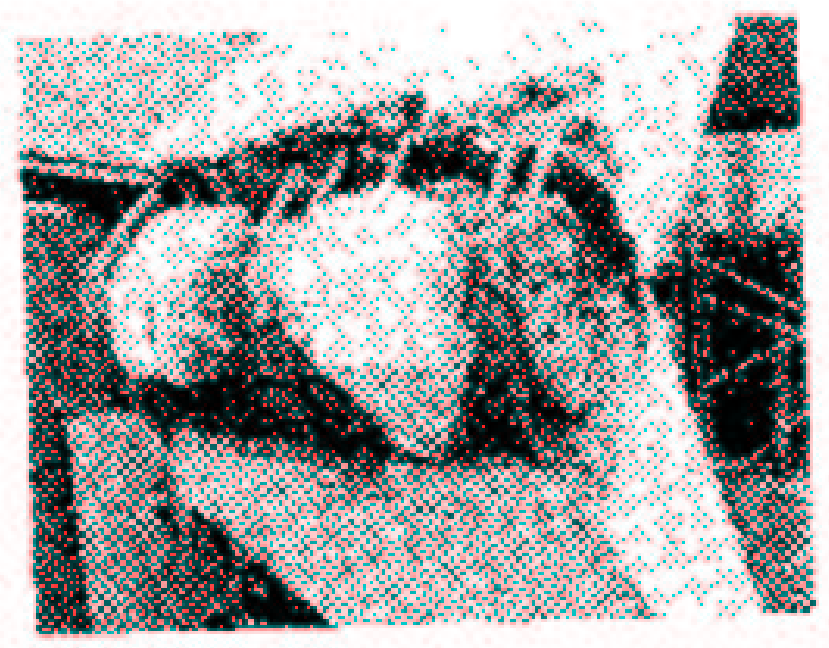

Figure 9: Typical tensile splitting failure, Johannson et al. [41] 


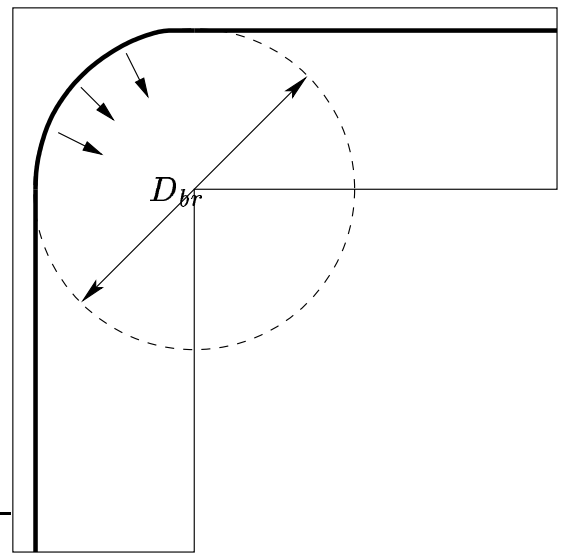

a)

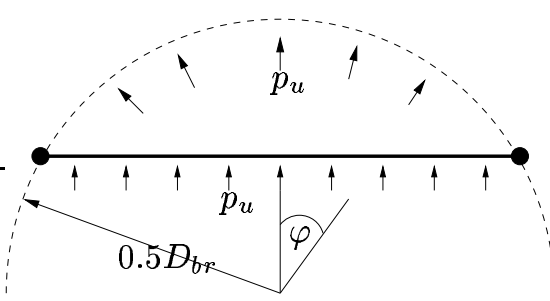

b)

Figure 10: Returning pressing

$p_{u, R}$ is reached, so that a very high stiffness in this direction is assumed. The maximum pressing $p_{u, R}$ is given according to experimental observation from [56] and [57] to

$$
p_{u, R}=\alpha_{u} f_{c t, s t} \sqrt{e_{R} D^{-1}}
$$

or

$$
p_{u, R}=\left(1.8-0.8 e^{-0.2 \eta}\right) \alpha_{u} f_{c t, s t} \sqrt{e_{R} D^{-1}}
$$

where $f_{c t, s t}$ is the splitting tensile strength of the concrete, $D$ is the diameter of the reinforcement, $e_{R}$ is the distance to the boundary and $\eta$ is given by

$$
\eta=\frac{A_{s q} f_{y}}{D l f_{c t, s t}}
$$

where $f_{y}$ is the yield strength of the steel and $A_{s q}$ is the area of the transverse reinforcement. The factor $\alpha_{u}$ is

$$
\alpha_{u}=\alpha_{u 0}\left(1-\frac{\epsilon_{r, r_{s}}}{\epsilon_{r, r_{s}, \max }}\right)
$$

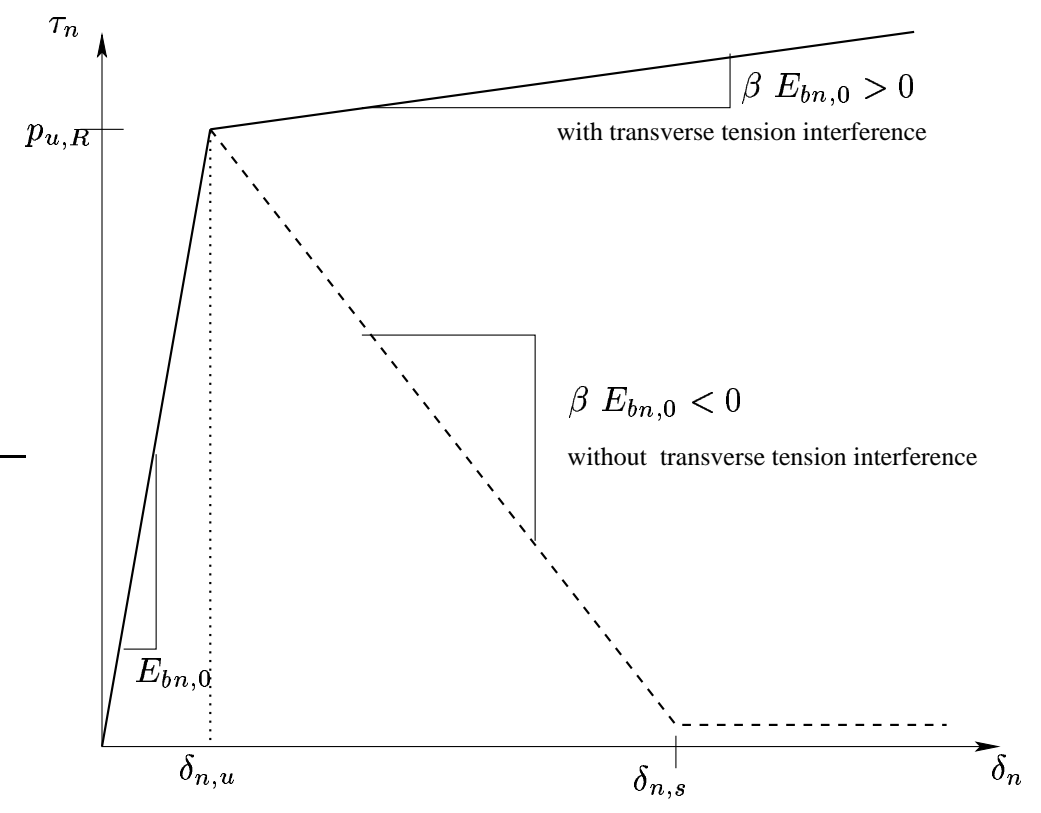

Figure 11: Bond behavior normal to the reinforcement 
Splitting tensile failure occurs when exceeding $p_{u, R}$. Depending on the transverse tension resistance, transverse pressing can occur or softening occurs in the bond model. Because of lack of experimental data, an approximation according to figure 11 is made. The reduction of the bond stiffness is controlled by a factor $\beta$. The radial stresses diminish the transverse tension resistance. In the case of a splitting failure, no transverse pressing occurs.

\section{RESULTS}

\subsection{Pull- and Pullout tests}

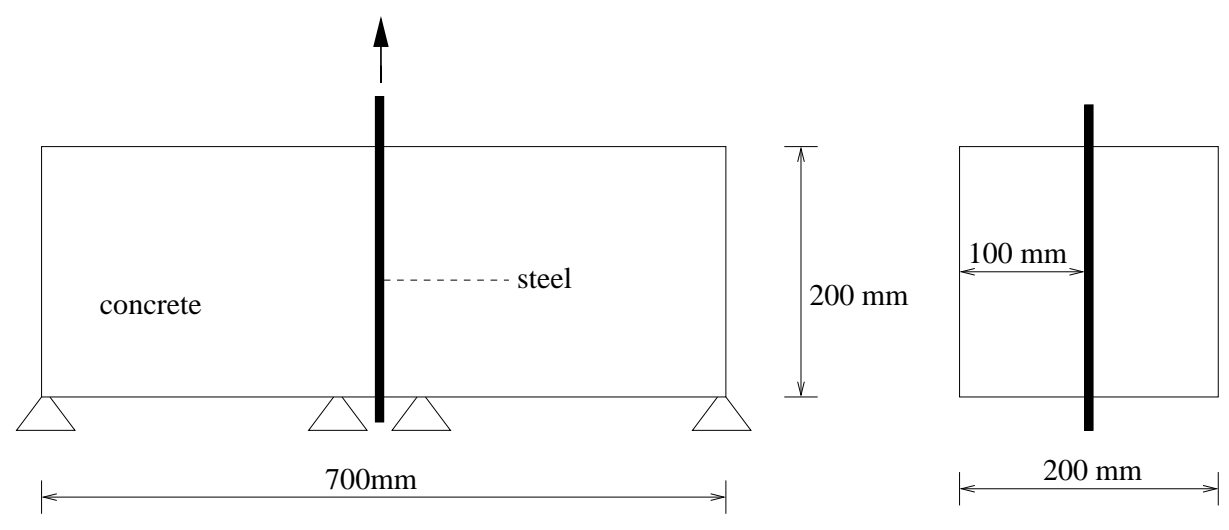

Figure 12: Test-setup for pullout test

A series of pullout test was performed by Idda [36]. The test setup is shown in figure 12. We discretized the problem simplified in 2D under plane stress assumptions. The reinforcement had a diameter of $16 \mathrm{~mm}$ and was modelled with beam elements. Particles were used for the concrete. The tensile strength for concrete was $f_{t}=$ $2.1 M P a$, compressive concrete strength $f_{c}=37 M P a$, Poisson ratio $\nu=0.2$, Young's modulus $E=28,000 M P a$ and fracture energy $G_{f}=100 \mathrm{~N} / \mathrm{m}$. The reinforcement had a Young's modulus of $210,000 M P a$, Poisson ratio $\nu=0.3$ and yield strength $\sigma_{y}=550 M P a$.

The reaction force-slip curve is shown in figure 13 for different numbers of particles (2000 and 8000) and shows a fair agreement to the experiments.

The necessity of a bond element is also shown at another simple pull test as shown in figure 14, see Eligehausen, Mayer [31]. In this case, a splitting failure occurs. The material parameters for concrete are: compressive strength $f_{c}=45 M P a$, tensile strength $f_{t}=2.12 M P a$, Poisson ratio $\nu=0.2$, Young's modulus $E=34,000 M P a$ and fracture energy $G_{f}=100 \mathrm{~N} / \mathrm{m}$. The parameters for the reinforcement are: Young's modulus 195GPa, yield strength $f_{y}=552 \mathrm{MPa}$ and tensile strength $f_{t}=646 \mathrm{MPa}$.

A crack pattern with and without bond model of the numerical simulation is shown in figure 15 . Too many cracks occur without a bond model. With bond model, the uniform crack pattern of the experiment can be reproduced pretty well. We would like to mention that the steel stresses remain below the yield strength of $552 M P a$.

\subsection{Three point bending of a reinforced concrete beam}

A series of three point bending tests of reinforced concrete beams were performed by Bosco and Debernardi [21]. We will focus on two of them, beam $T 5 A 1$ and $T 6 A 1$. The test setup and the dimensions of these two experiments can be found in figure 16. Beam $T 6 A 1$ has a higher degree of reinforcement at the bottom than beam $T 5 A 1$. Hence, beam $T 5 A 1$ failed due to plastic flow of the lower reinforcement and beam $T A 6$ due to the failure of the concrete compression zone in the middle of the specimen.

In the computation, we used symmetry conditions and modelled only half of the structure. Beam elements were used for the stirrups and for the longitudinal reinforcement. Plane stress conditions are assumed. We will show the results for approximately 8200 particles but we also performed computations with finer and coarser particle arrangements. The results did not show mesh dependence. We will show the absence of mesh dependence for another example in section 8.4.

The concrete material parameters are compressive strength $f_{c}=-32.0 M P a$, tensile strength $f_{t}=2.5 M P a$, Poisson ratio $\nu=0.22$, Young's modulus $E=28,000 M P a$ and fracture energy $G_{f}=100 \mathrm{~N} / \mathrm{m}$. The reinforcement 


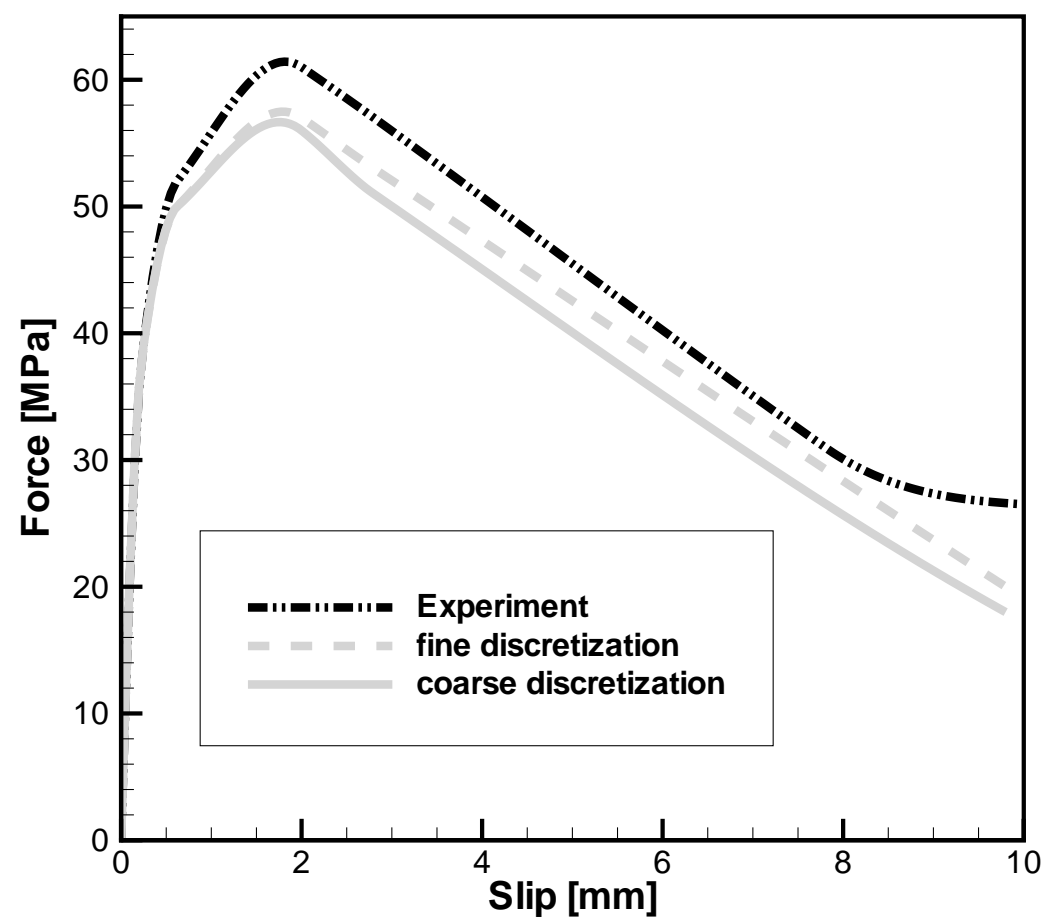

Figure 13: Force-slip curve for the pullout test by [36]

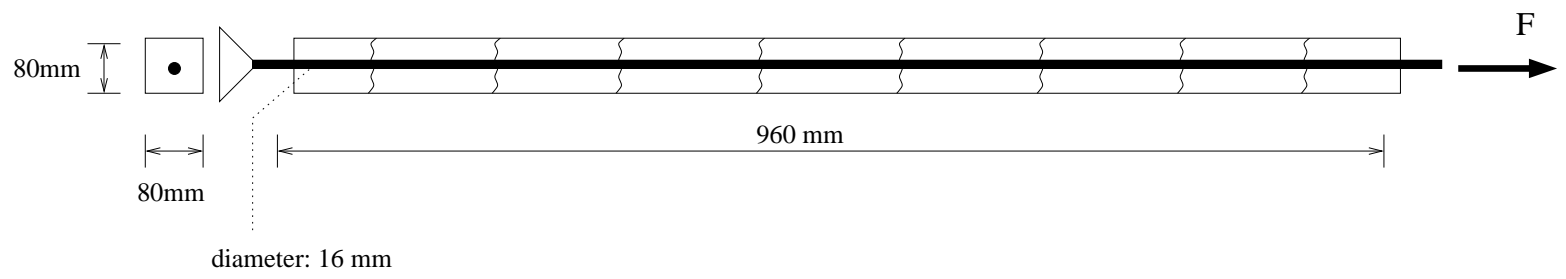

Figure 14: Test-setup for pull test by [31]

has a yield strength of $f_{y}=587 M P a$, tensile strength $672 M P a$, Young's modulus $E_{s}=200,000 M P a$.

Figure 17 shows the crack pattern at time of fracture for the computations. The computational crack pattern agrees well with the crack pattern of the experiment (for both experiments), see [21]. Note that the crack curves nicely even for such a coarse discretization which is not obtained for some smeared crack models using finite elements and a finer discretization, see e.g. Akkermann [1].

We also plotted the compressive damage. For beam $T 6 A 1$, a high damage compression zone can be observed, which causes a rapid decrease in the slope of the load-mid displacement curve shown in figure 18. Beam $T 5 A 1$ fails due to the plastic flow of the lower reinforcement. The damage in the compressive zone is negligible compared to experiment $T 6 A 1$.

The load mid-displacement curves are shown in figure 18. The agreement between the experiments and the computations is excellent. Also the maximum mid displacement due to failure is replicated well.

\subsection{Failure of frame corners}

A series of experiments on the failure of frame corners subjected to a positive and negative moment load were performed by Akkermann [1]. The arrangement of the reinforcement is more complicated than in the other example. We will focus on three experiments as shown in figure 19 which showed three different failure mechanisms. 


\section{without bond model}

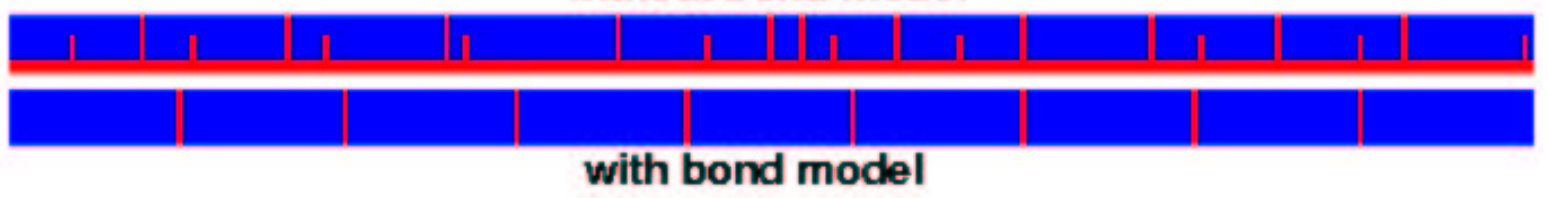

Figure 15: Crack pattern of the pull test by [31] with and without bond model

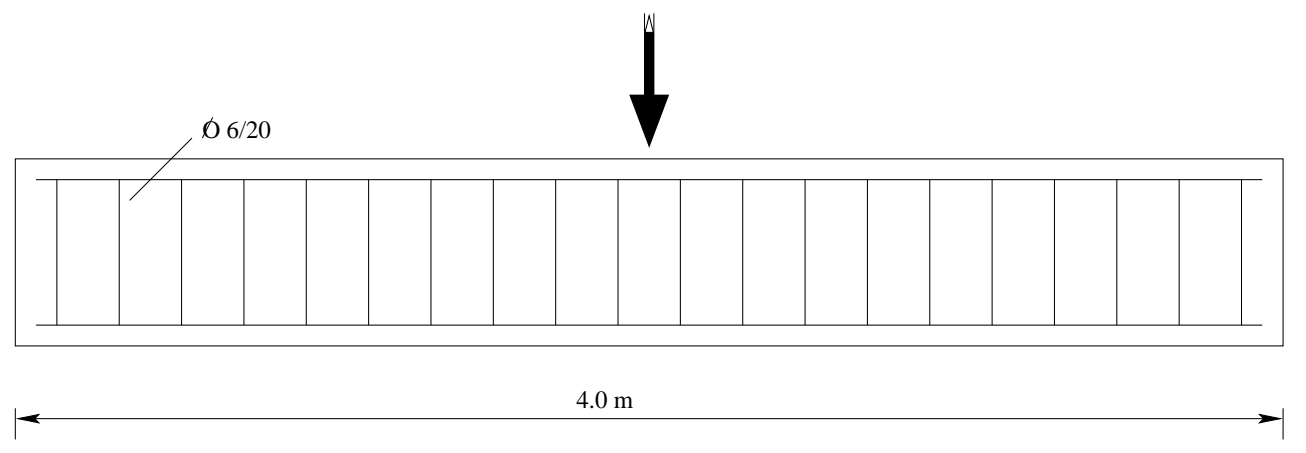

T5A1

T6A1

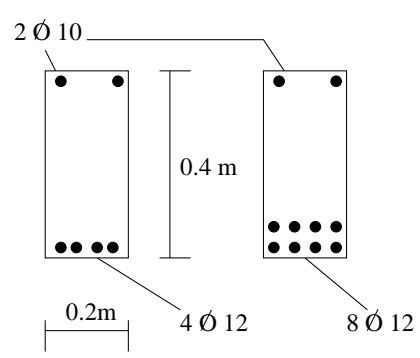

Figure 16: Test setup of the Bosco beam [21] T5A1 and T6A1

The concrete tensile strength in these experiments was tested at cylindrical specimen of $150 \mathrm{~mm}$ diameter and $300 \mathrm{~mm}$ height and varied between 2.5 MPa and 2.8 MPa. The Young's modulus was between 24,000 $M P a$ and 28,000 MPa, the compressive strength between $25 \mathrm{MPa}$ and $33 \mathrm{MPa}$ and the fracture energy between $100 \mathrm{~N} / \mathrm{m}$ and $124 \mathrm{~N} / \mathrm{m}$. With a given fracture energy and a given critical tensile stress and assuming a linear relation between traction and crack opening, the slope of the cohesive model follows automatically. Different kind of reinforcements were used as shown in figure 19. The yield strength for the different steels ranged from $510 M P a$ to $603 M P a$ and the Young's modulus from 180,000 $M P a$ to 205,000 $M P a$. More details about the experiments can be found in Akkermann [1].

Frame corner $F C 1$ was loaded with a positive moment, see figure 19a, and failed due to concrete failure, meaning by pulling of the corner. The bond behavior is of minor importance in this example. Hence, we connected the reinforcement to the concrete rigidly. We used a structured equidistant particle arrangement and show the results for approximately 12,600 particles. Similar results were obtained with approximately 6,000 particles but the influence of the discretization will be discussed for frame corner $F C 2$. The diameter $D_{b r}$ (see figure 10) plays an important role in the crack pattern and failure mechanism of the structure and is $30 \mathrm{~cm}$ for $F C 1$.

Frame corner $F C 2$ was loaded with a negative moment and a splitting tensile failure occurred. In this example, the interaction between concrete and the reinforcement is crucial, so we used the bond model explained before. We ran with two different meshes, one with a particle separation of $1 \mathrm{~cm}$ and another one with a particle separation of $1.6667 \mathrm{~cm}$, with 10,000 and 3,700 particles, respectively. The diameter $D_{b r}$ is $50 \mathrm{~cm}$.

For frame corner $F C 3$, the bond behavior is also important. Frame corner $F C 3$ was also loaded with a negative moment and failed due to a splitting failure. Since the concrete geometry is equivalent to frame corner $F C 1,12,600$ particles were used. For all frame corners, plane stress conditions are assumed. The diameter $D_{b r}$ is the same as for frame corner $F C 2$.

Figure 20a shows the crack pattern of the computation for structure $F C 1$. The diagonal crack causes the failure of the structure. Several small cracks at the inside of the frame corners are observed which matches well with the experimental data. The load displacement curves from the experiment and computation are illustrated in figure 21a and show a good agreement.

Frame corner $F C 2$ failed due to a splitting tensile failure. This was also reproduced by the computation. The crack pattern is shown in figure $20 \mathrm{~b}$ and matches well with the experiment. Many cracks at the outside of the frame corner are observed. Figure 21a shows the load displacement curve. A sudden drop in the displacement curve can be observed which is caused by the sudden splitting tensile failure. Note that we were able to reproduce this failure mode without a non-local approach. Akkermann [1] reported that without non-local models, no splitting tensile 


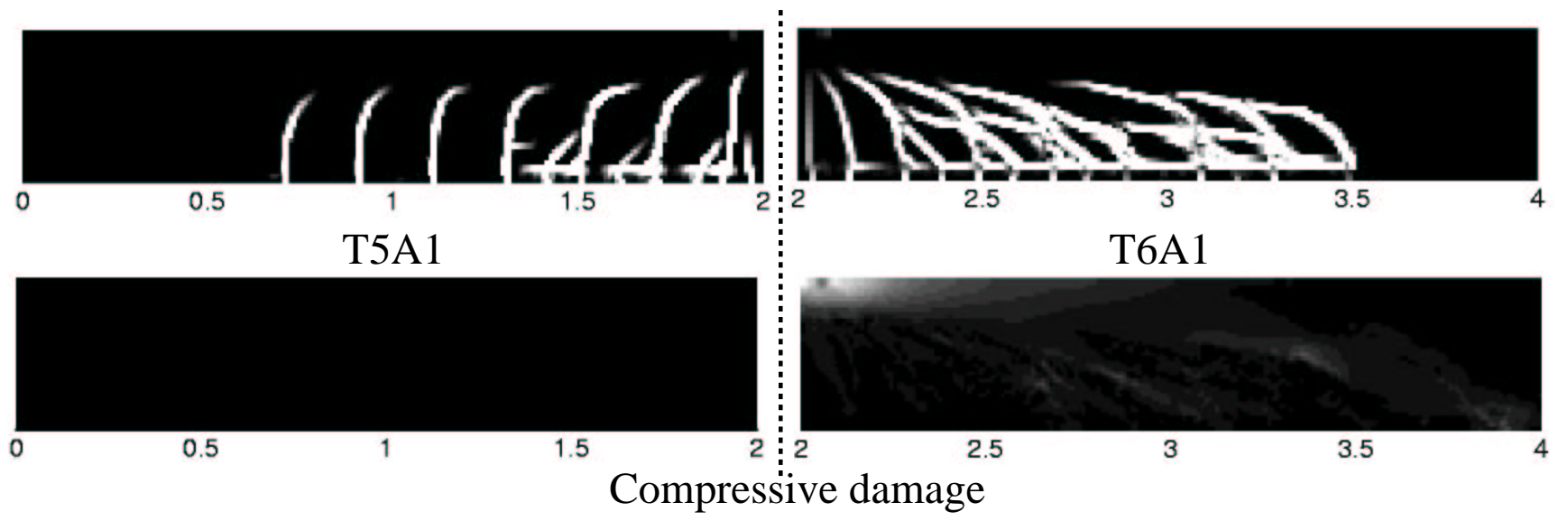

a) numerical results
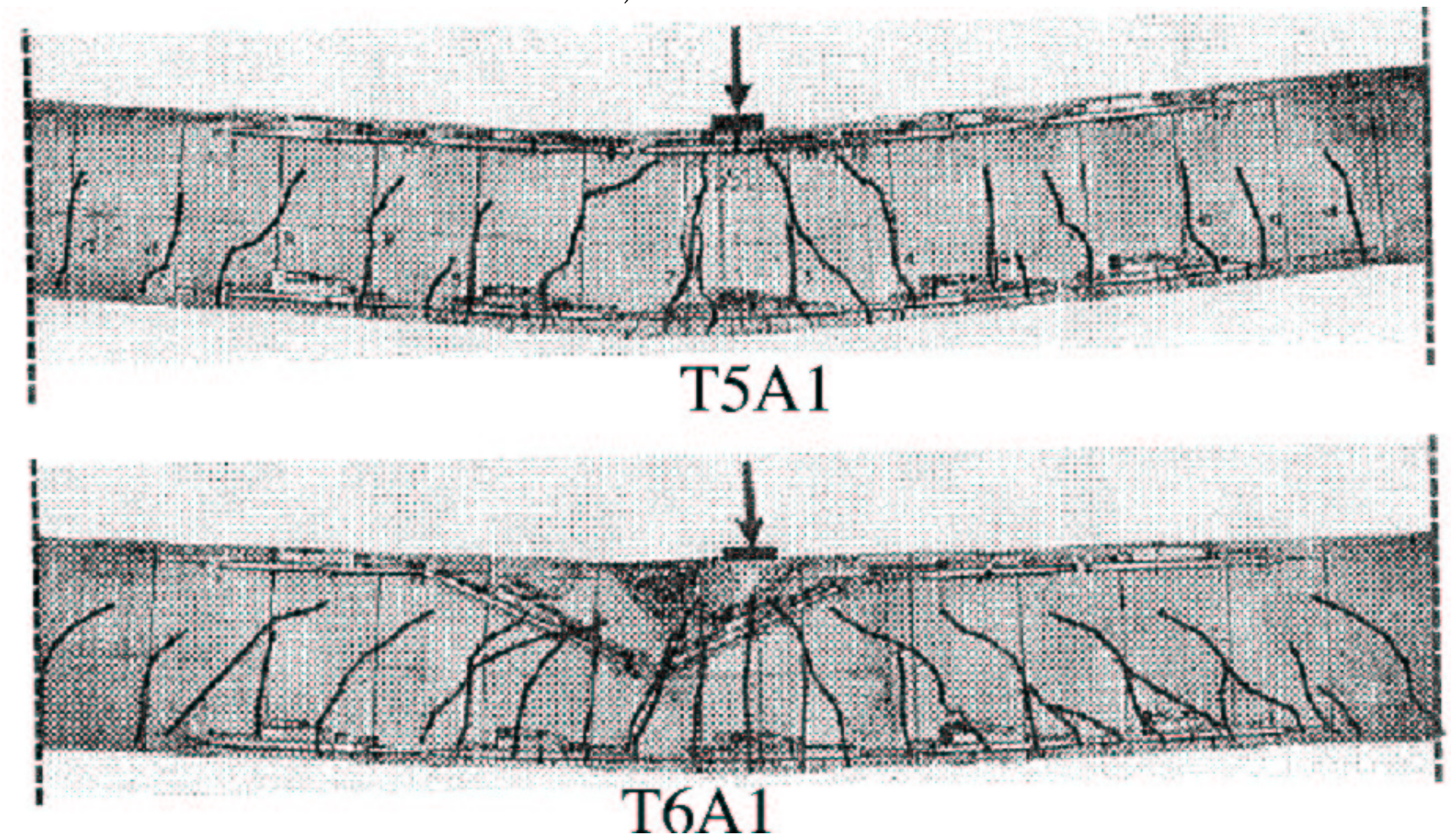

b) experimental results

Figure 17: a) Computed crack pattern of the experiment $T 5 A 1$ (left) and $T 6 A 1$ (right) and compressive damage (lower figures) compared to b) the experimental results by [21] 


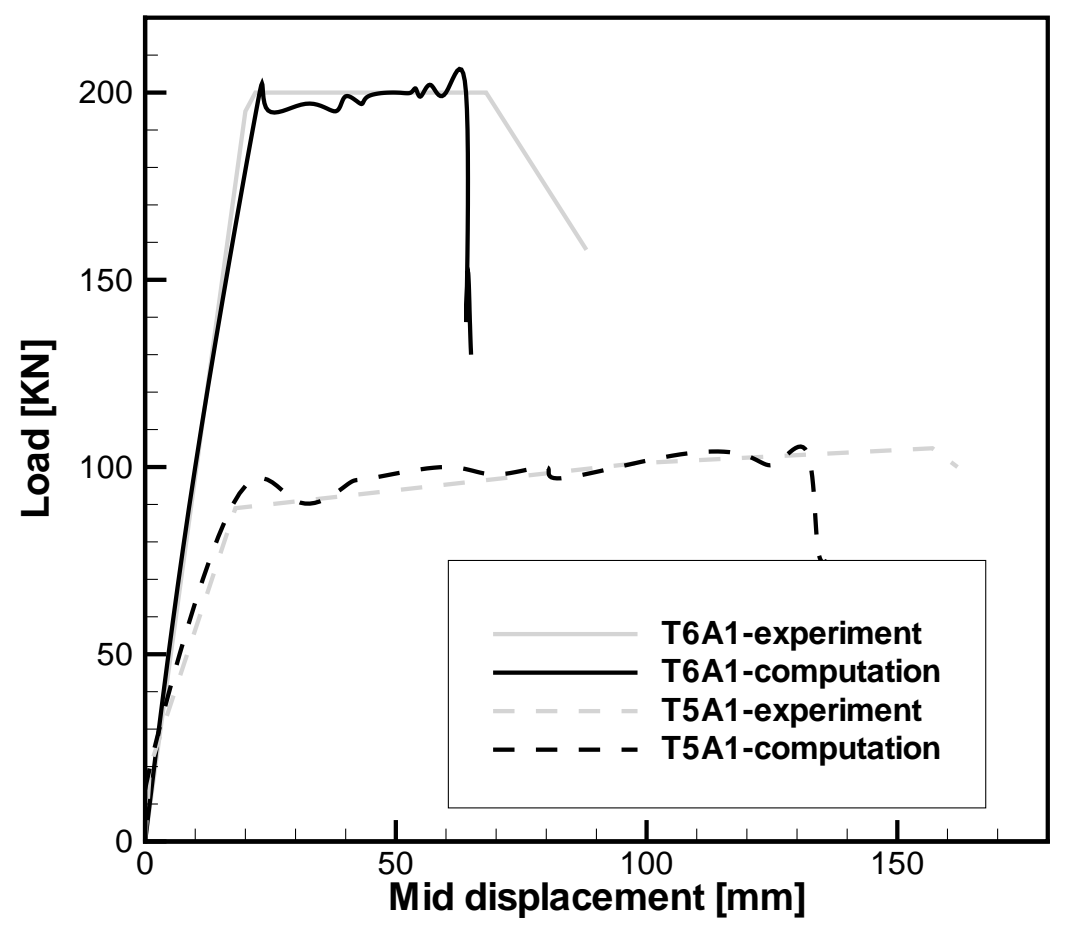

Figure 18: Load-mid displacement curves of the beams $T 5 A 1$ and $T 6 A 1$ of the experiment compared to the computation 


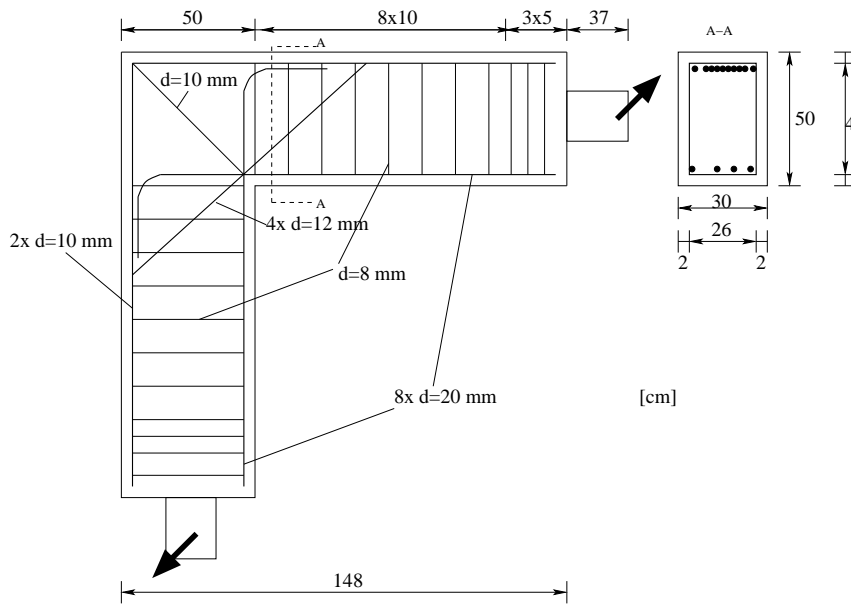

a)

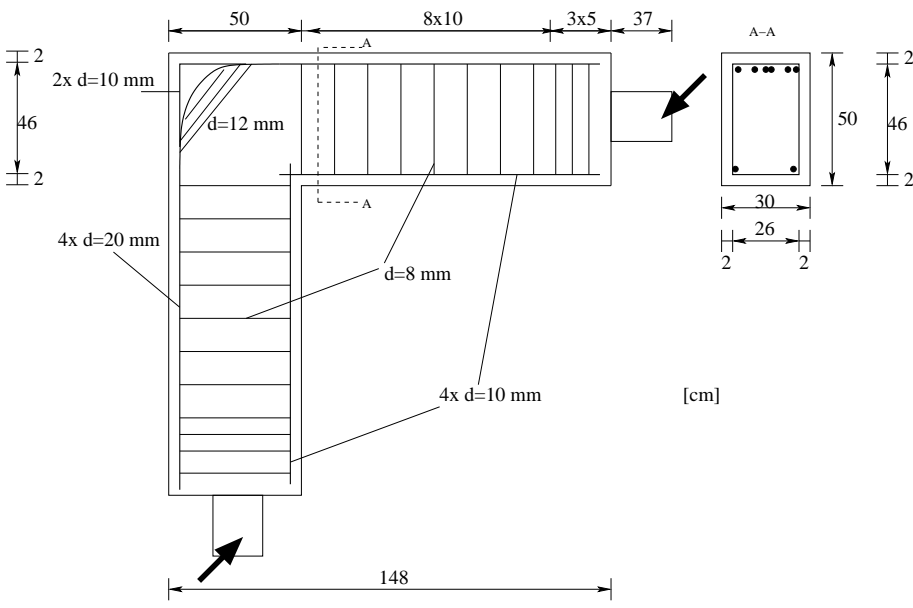

b)

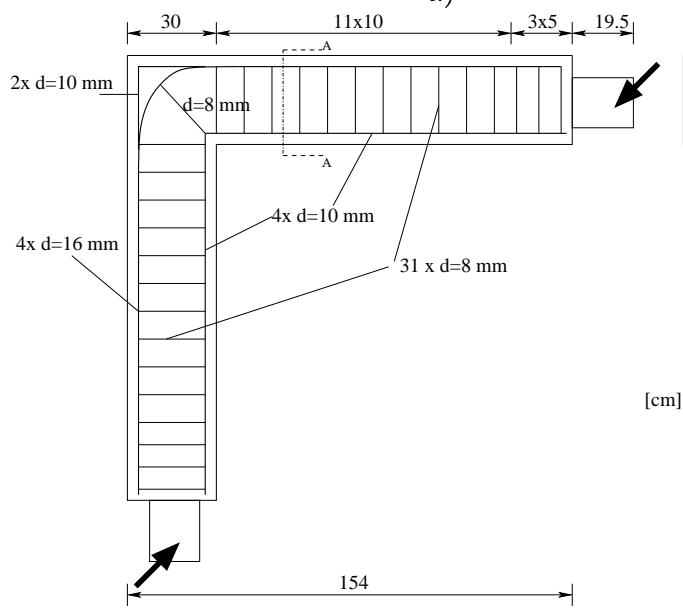

c)

Figure 19: Test setup of frame corner a) $F C 1$, b) $F C 3$, c) $F C 2$

failure could be obtained in a finite element analysis using a smeared crack approach. With meshfree methods, the non-locality can be enhanced by a large support. The influence of the mesh-refinement is negligible.

Figure 20c shows the crack pattern of frame corner $F C 3$ which failed due to a splitting failure. Figure 21b compares the computational and experimental load displacement curve. They are in good agreement.

\subsection{Prestressed concrete beams}

A series of prestressed concrete beams of different cross section were performed by Eibl et al. [30]. We consider two prestressed concrete beams. The first one is of rectangular cross section. The test setup and the dimensions of the beam are illustrated in figure 22. The beam was prestressed with two tension wires of $7 \mathrm{~mm}$ diameter. The upper one was prestressed with a force of $26.25 K N$, the lower one with a force of $11.25 K N$. The beam failed in bending because of the plastic flow of the lower reinforcement. An elastoplastic constitutive model with isotropic hardening was used for the reinforcement.

The test setup for the second beam is similar to the first one and can be found in figure 23. In contrast to the first beam, the second one is an I-cross section. The beam has two tension wires of $12 \mathrm{~mm}$ diameter at the lower flange which were prestressed with a force of $80 \mathrm{KN}$. The diameter of the upper reinforcement is $10 \mathrm{~mm}$. Because of the lack of a stirrup reinforcement, the beam failed due to a combined shear/pullout failure.

Both beams are discretized in two dimensions. Plane stress conditions are assumed. We used the symmetry and modelled only half of the beam. Different refinements were tested. We will present the results for approximately 2200 and 8650 concrete particles.

The concrete material parameters for both beams are Young's modulus 29, 000MPa, tensile strength 2.8MPa, compressive strength $44 M P a$. The fracture energy was $G_{f}=86 M N / m$. Also the parameters for the prestressing 


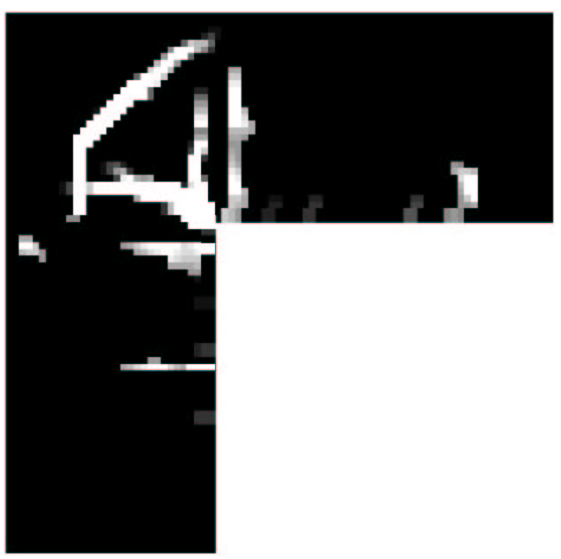

a) $F C 1$
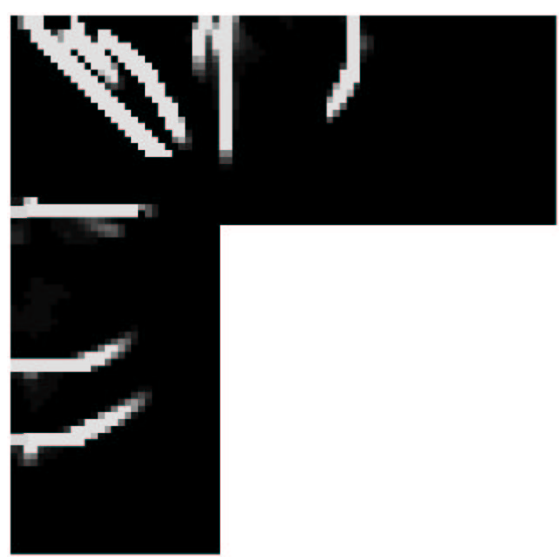

b) $\mathrm{FC} 2$

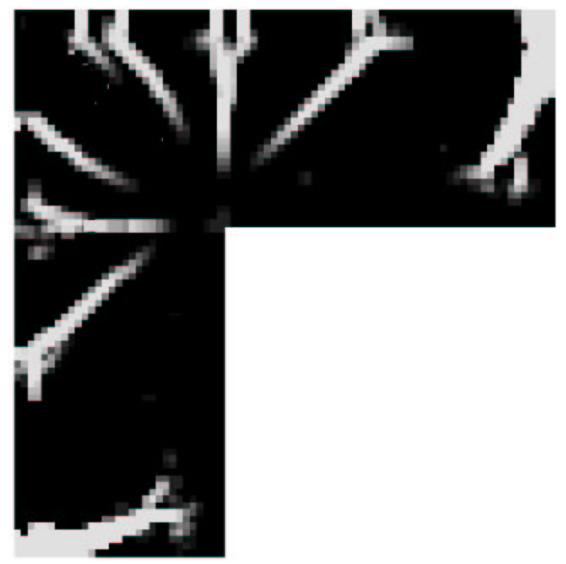

c) $F C 3$

Figure 20: Crack pattern of frame corners a), b), c) from the computations

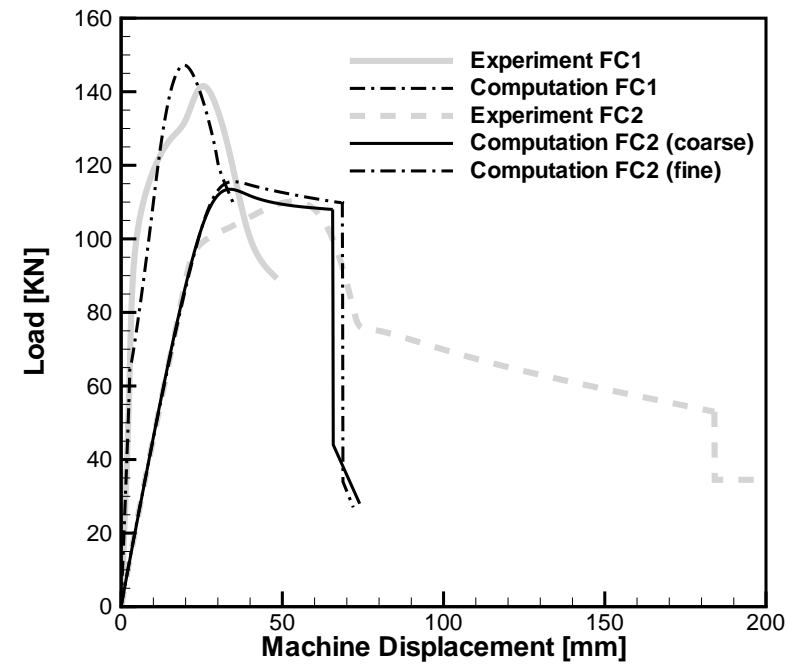

a)

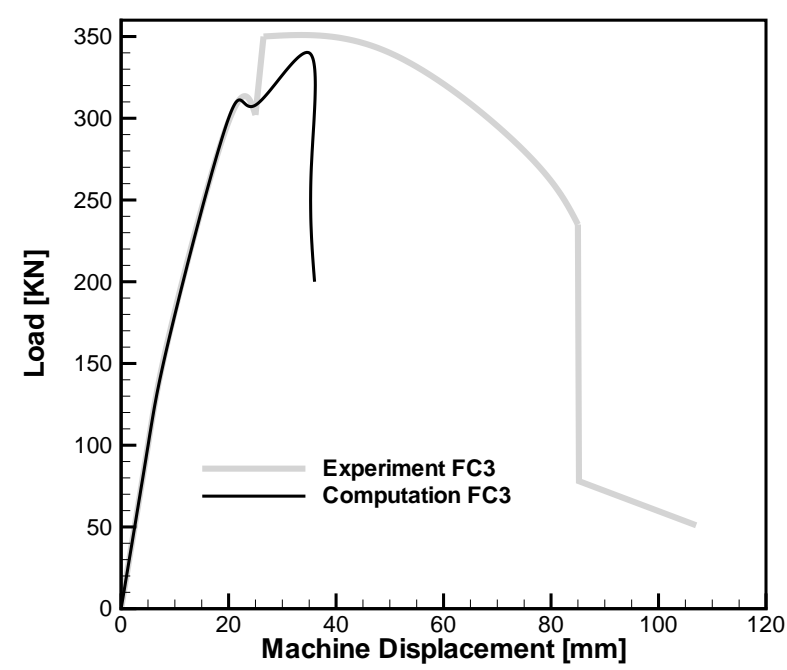

b)

Figure 21: Load Displacement of a) $F C 1$ and $F C 2$ b) $F C 3$

wires are the same with Young's modulus 205,000 $M P a$, yield strength $f_{y}=1420 M P a$ and tensile strength $f_{t}=1570 M P a$. For beam II, the Young's modulus for the upper not-prestressed reinforcement is 210,000 $M P a$ and the yield strength $500 \mathrm{MPa}$.

The prestressing is modelled via a temperature loading of the tension wires so that the stresses measured in the experiments are obtained. In other words, the tension wire is shortened by cooling them. The strains are computed by $\epsilon=\alpha_{t} \Delta T$ where $\alpha_{t}$ is the thermal expansion coefficient which is $1 \cdot 10^{-5} / C$ for steel and $\Delta T$ is the temperature difference, which is negative in our case. The contraction of the tension wire transmits the prestressing forces in the concrete. For the first beam, we applied a temperature of -315 Kelvin for the lower r-bar and -125 Kelvin for the upper r-bar so that the measured stresses according to the experimental data was obtained. For the second beam, the temperature was -315 Kelvin. Note that the shortening of the concrete beam due to the prestressing forces has to be taken into account. This effect also relaxes the prestressing and decreases the stresses in the prestressing wires.

Figure 24 compares the computed crack pattern with the experiment for beam I. The calculation exhibits more cracks than the experiment but the agreement is still pretty good. Note that we used symmetry, so only half of the beam is discretized. The cracks in the zone where the transverse force is zero are more or less straight. Cracks which evolve in the zone where the transverse force is constant start to curve nicely. 


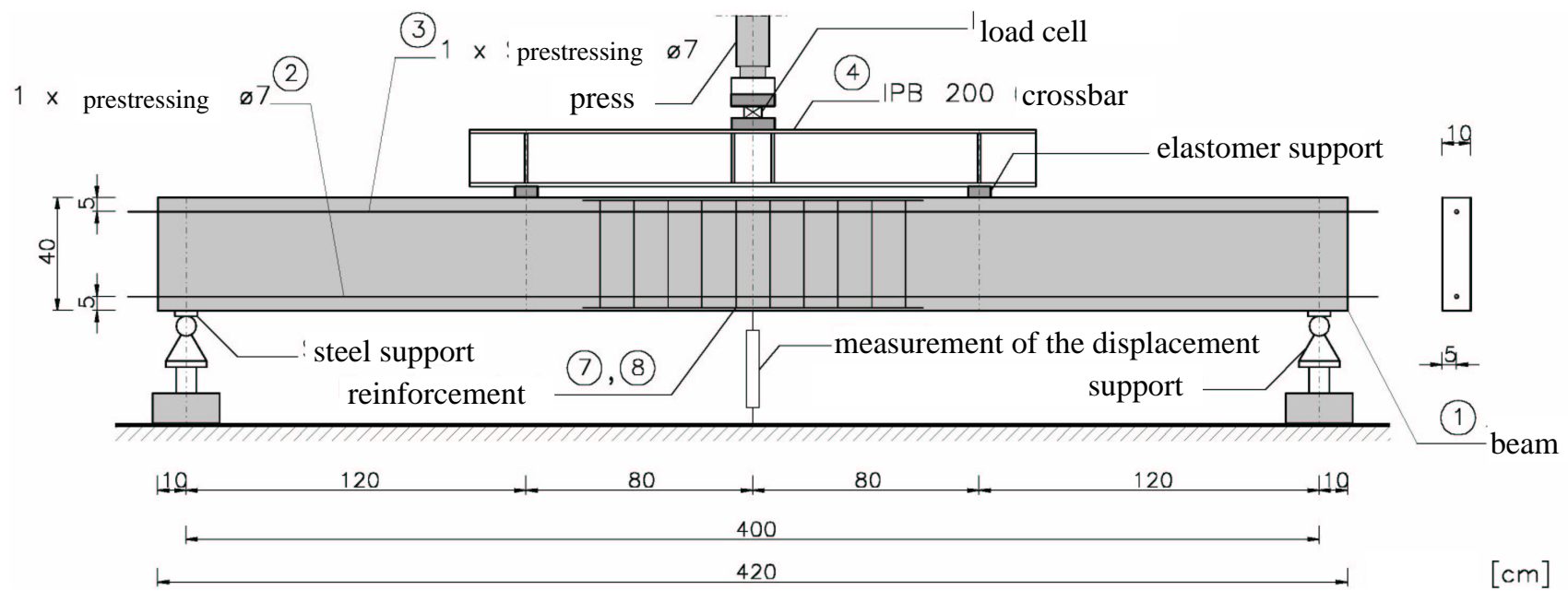

Figure 22: Test setup of beam I

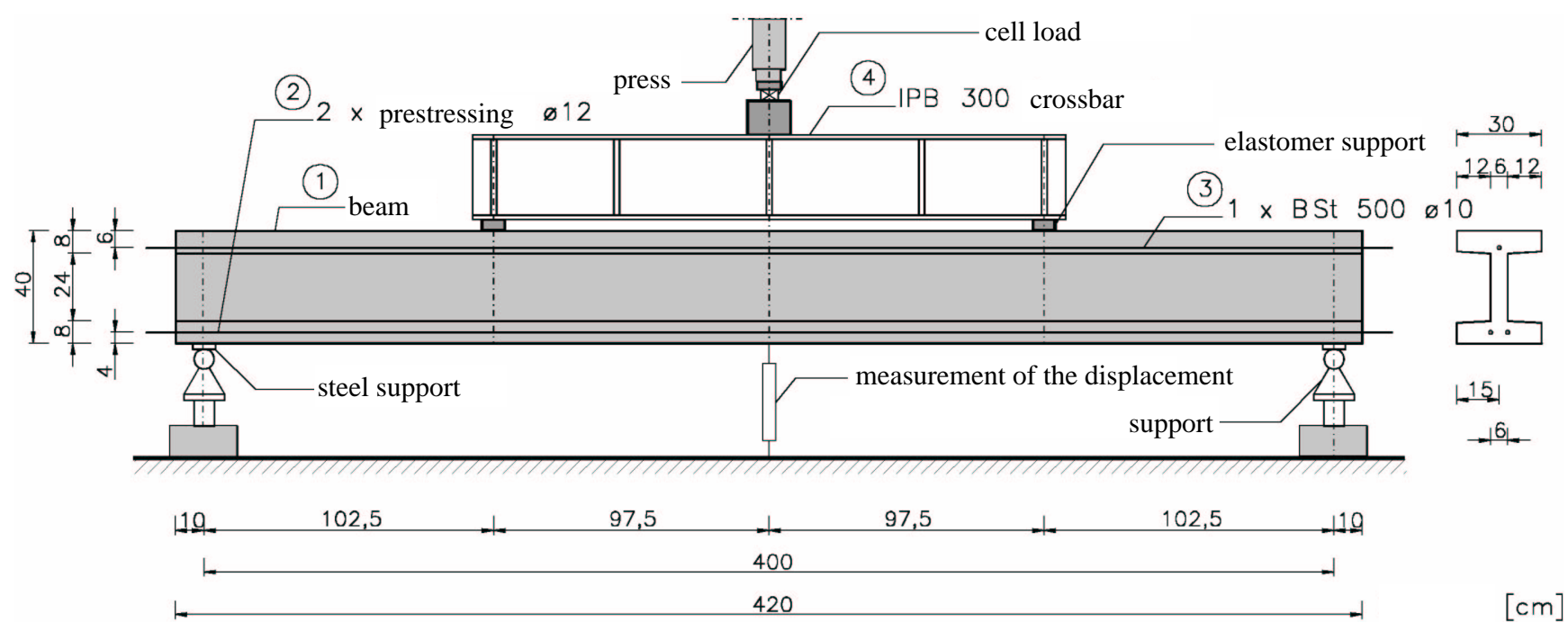

Figure 23: Test setup of beam II 

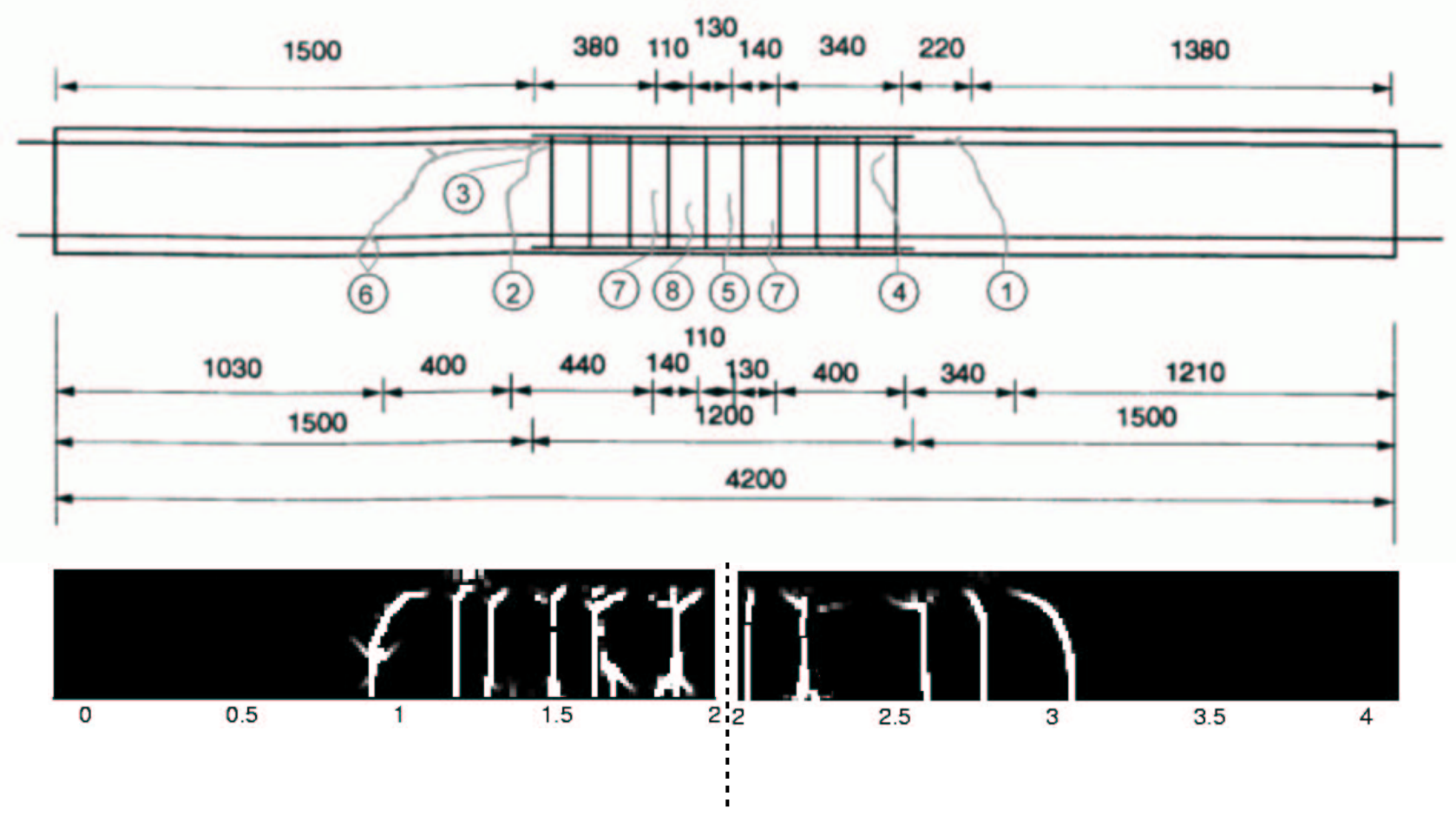

Figure 24: Comparison of the crack pattern of the experiment with the simulation for beam I

The beam failed due to plastic flow of the lower reinforcement which is replicated also by the numerical analysis. The experimental and numerical load displacement curves are illustrated in figure 25a and show a very good agreement. Both the slope and the mid displacement due to failure is reproduced well. In figure 26, the stresses in axial direction of the lower prestressing wire is shown. As can be seen, the tensile strength of $1570 \mathrm{MPa}$ of the steel is exceeded and the prestressing fails. It can also be seen that the results do not depend on the discretization.

Figure 28 shows the crack pattern of the experiment and the computation for beam II; a picture of the broken beam is shown in figure 27. The shear crack in the experiment is closer to the support than in the computation. In the experiment a combined shear/anchorage failure was observed. The simulation was able to predict only the shear failure. The shear crack continues to grow along the transition between the flange and the web instead of causing the bond failure. We think that the anchorage failure cannot be reproduced because of the two dimensional discretization, i.e. due to the large changes in the thickness between the flange and the web. A three dimensional simulation would probably overcome this difficulty.

The load mid-displacement curves of the experiment and two computations with different numbers of particles are compared in figure 25b and show fair agreement; we did not capture the sudden failure of the beam at about $7 \mathrm{~mm}$ displacement. The steel stress and the bond stresses across the lower reinforcement are shown in figure 29 and 30. The steel stresses are below the yield strength and no plastic strains occur which matches well with the observation in the experiment. Observing the bond stresses, it can be recognized that no bond failure occurs but the curves look quite reasonable.

\section{SUMMARY AND DISCUSSION}

We have described a coupled particle/finite element method based on Lagrange multipliers. We extended the coupling method to nonlinear problems and deformable interfaces, i.e. relative displacements between the different domains are allowed. The coupled particle-finite element exploits the advantages of meshfree methods for modelling fracture. The advantage of meshfree methods is that the crack path does not depend on the mesh alignment. Also the non-local character in interpolation can be advantageous.

The coupling method is applied to reinforced concrete structures where the reinforcement is modelled with finite elements and the concrete with particles. The meshfree discretization is not influenced by the "mesh"-constraint which promises better results in the crack path. 


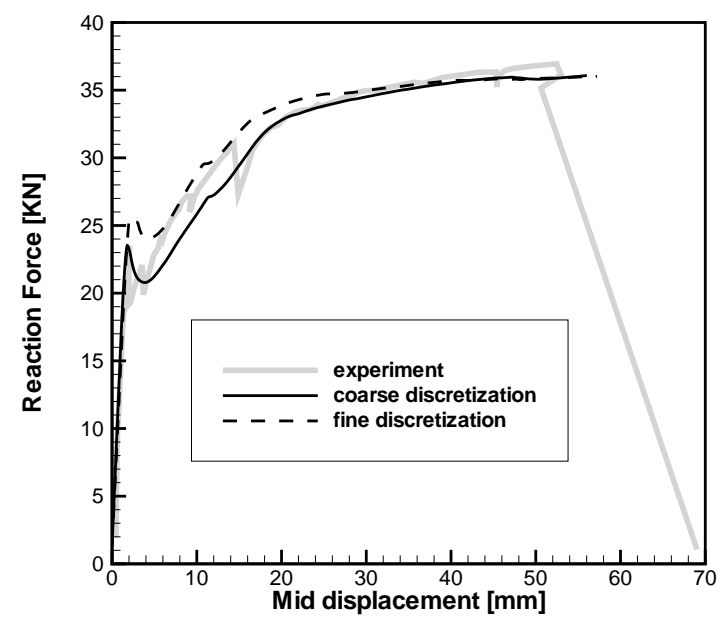

a)

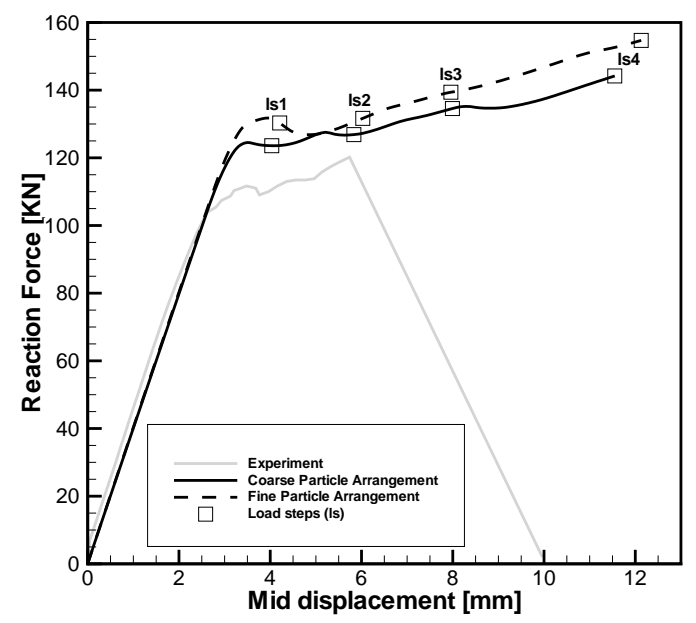

b)

Figure 25: Load mid displacement curve in dependence of the number of particles

A bond model is proposed which can model the basic bond failure mechanisms, a pullout and a splitting failure. Though the discretization is only two-dimensional, the bond model is extended to capture three-dimensional effects.

The method is applied to several fracture problems of reinforced concrete structure where the reinforcement is modelled with finite elements and the concrete with particles. The computational results show good agreement to experimental data. Good crack pattern especially in terms of curvature are obtained. 


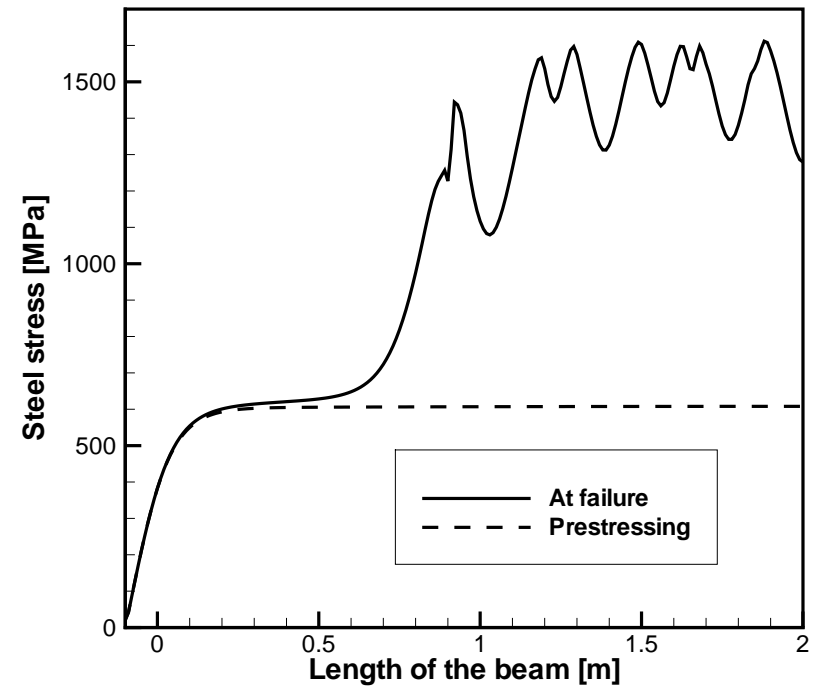

a)

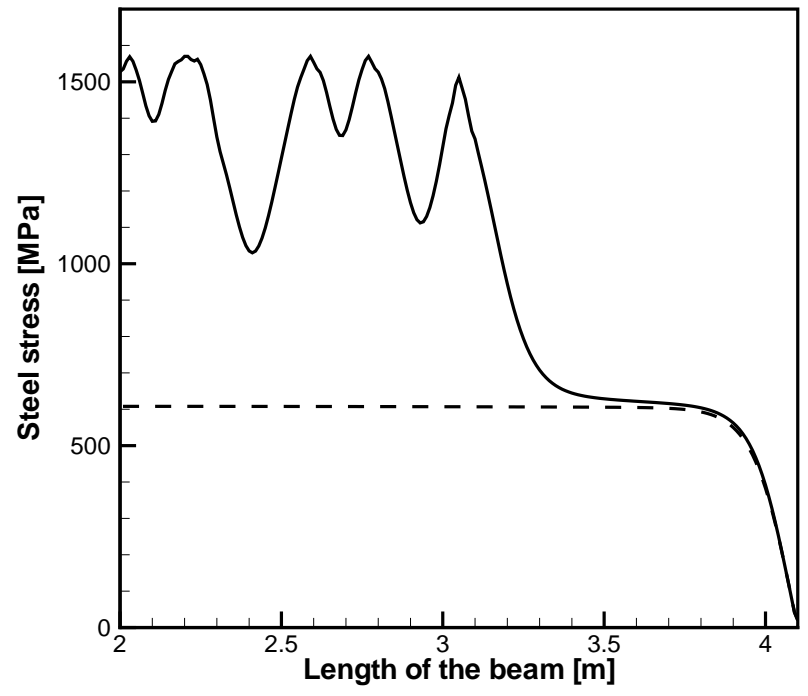

b)

Figure 26: Steel stresses of the computation for beam I, a) coarse discretization b) fine discretization

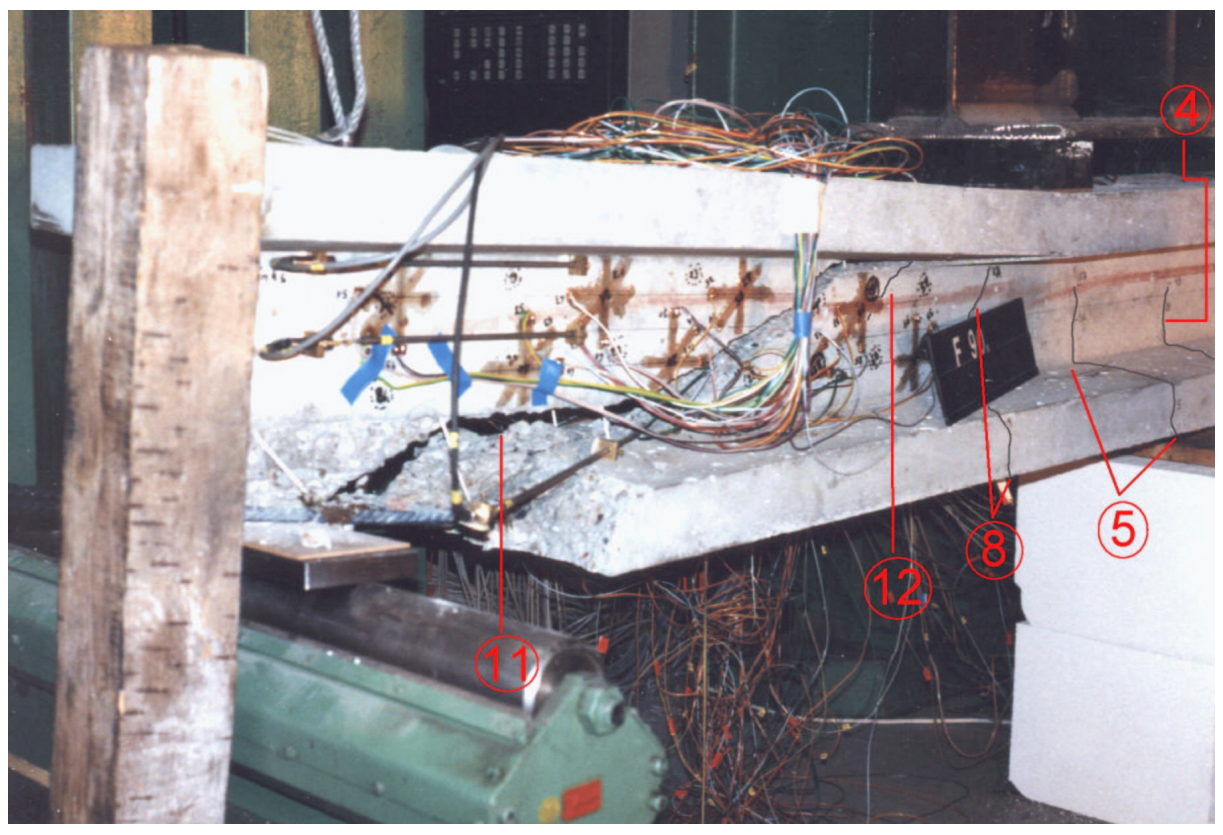

Figure 27: Picture of a prestressed concrete beam with I cross section after the experiment 

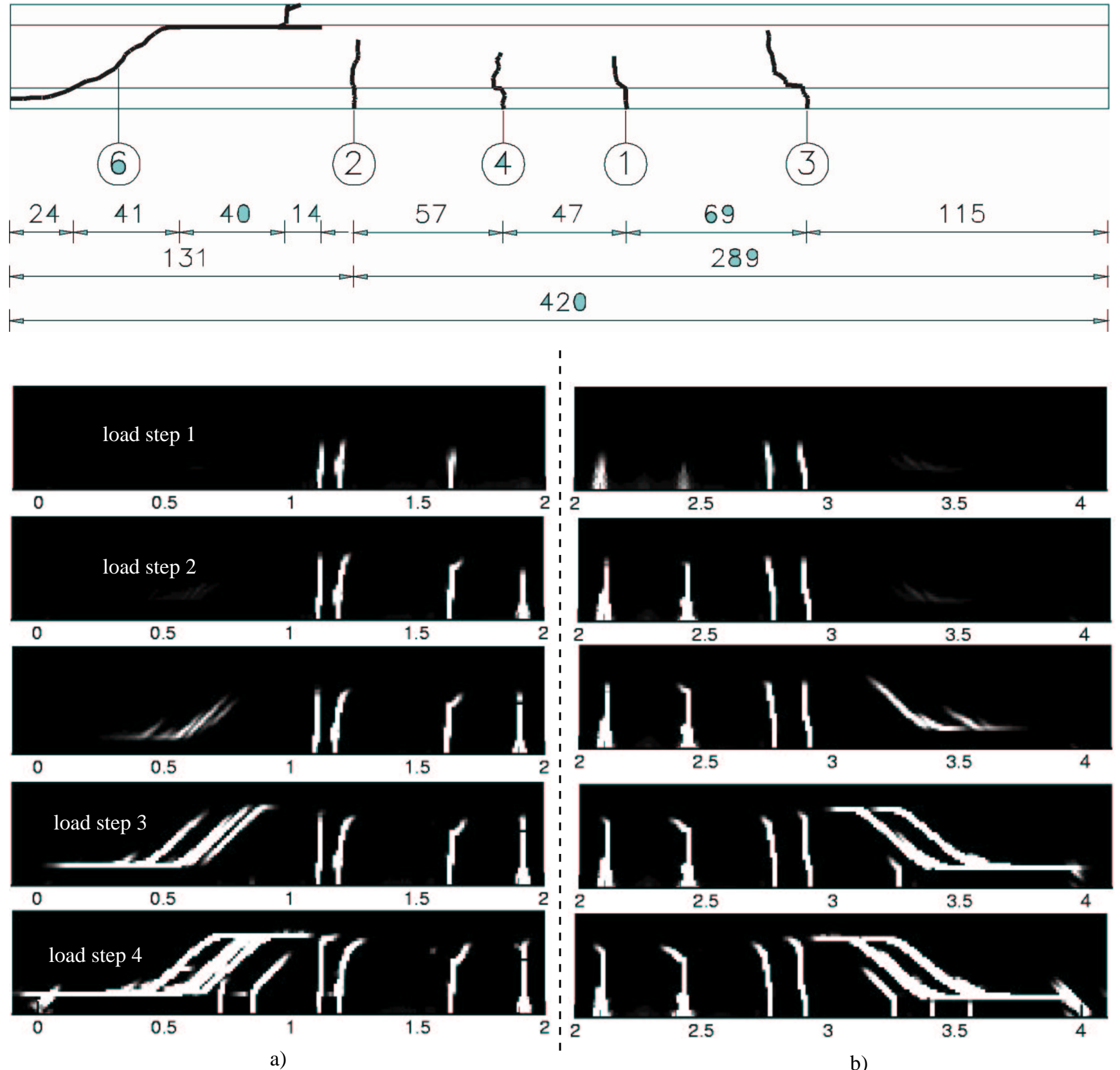

b)

Figure 28: Comparison of the experimental crack pattern with the numerical results for beam II at different load steps for a) 2200 particles and b) 8650 particles 


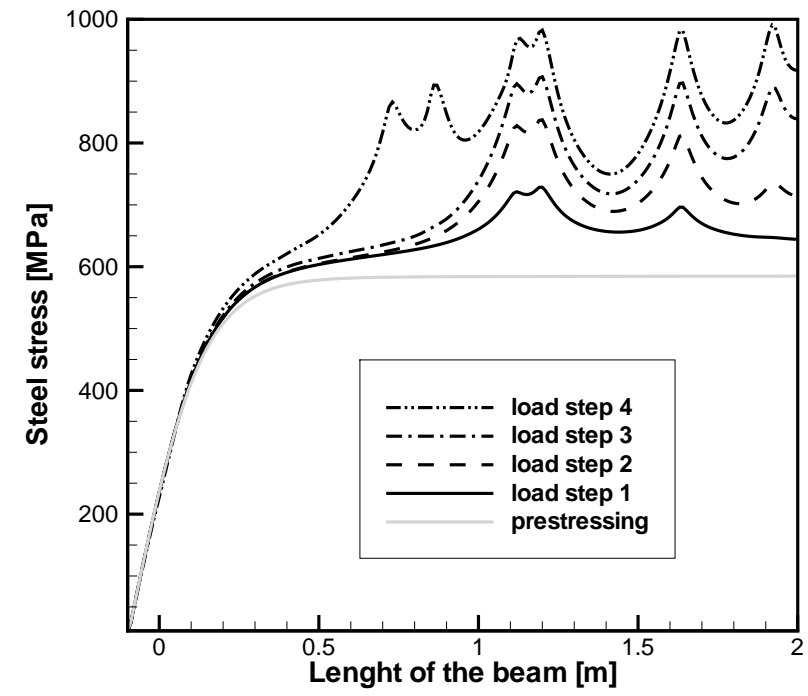

a)

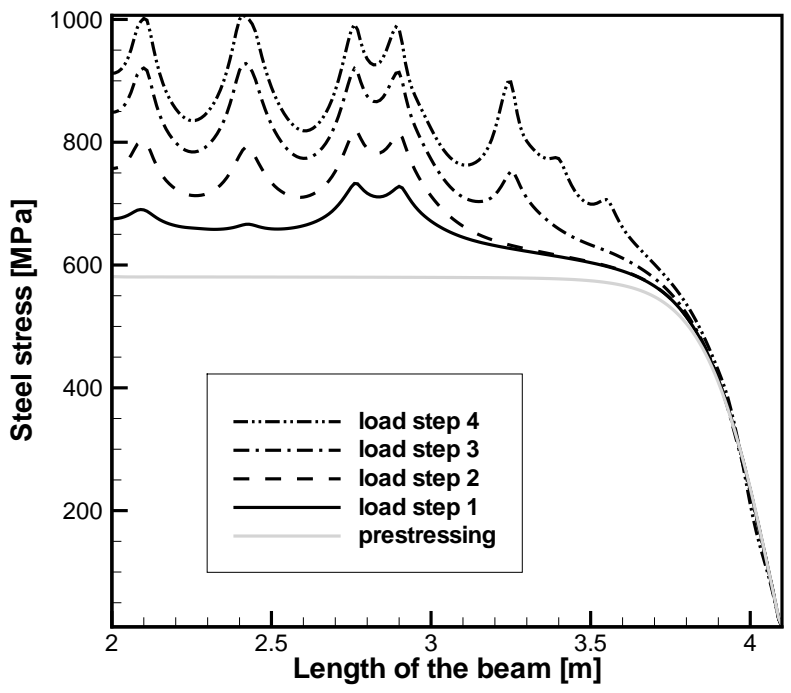

b)

Figure 29: Steel stresses of the lower reinforcement at different load steps for beam II, a) 2200 particles and b) 8650 particles

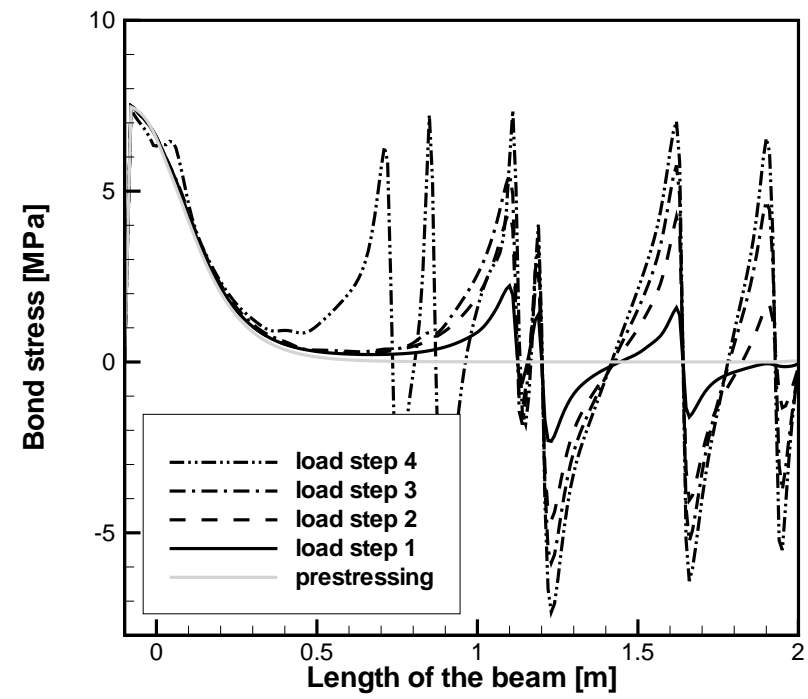

a)

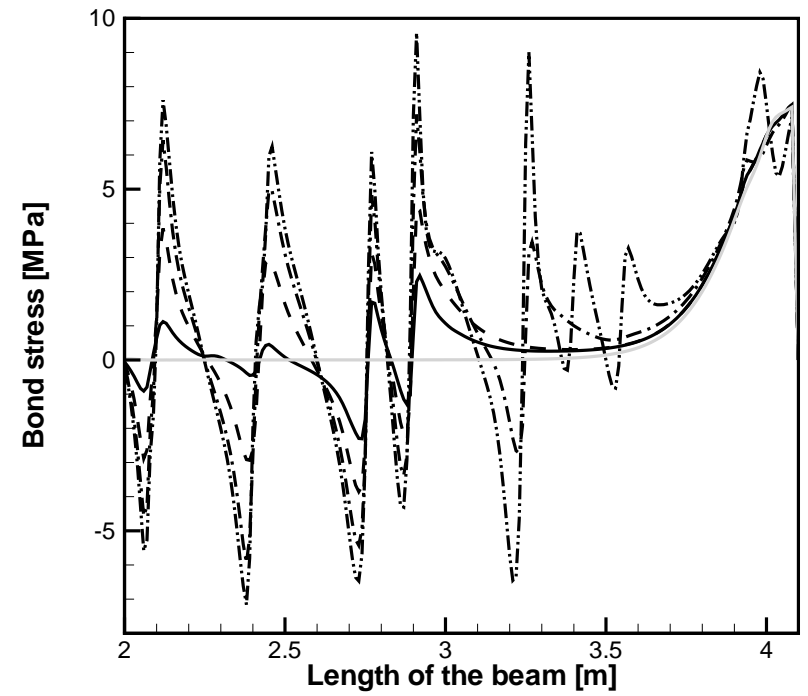

b)

Figure 30: Bond stresses across the lower reinforcement at different load steps for for beam II, a) 2200 particles and b) 8650 particles 


\section{References}

[1] Akkermann J.: Rotationsverhalten von Stahlbeton-Rahmenecken, Dissertation, 2000, Institut fuer Massivbau und Baustofftechnologie, Heft 39, Universitaet Karlsruhe

[2] Arrea M., Ingraffea A.R.: Mixed-mode crack propagation in mortar and concrete, Rep. No. 81-13, Dept. of Struct. Eng., Cornell University Ithaka, N.Y., 1982

[3] Attaway S.W., Heinstein M.W., Swegle J.W.: Coupling of Smoothed Particle Hydrodynamics with the Finite Element Method, Nuclear Engineering and Design 150, 1994, Post-SMIRT Impact IV Seminar Berlin

[4] Babuska I., Melenk J.M.: The partition of unity finite element method, University of Maryland, Technical Note $\mathrm{BN}-1185,1995$

[5] Bazant Z.P., Belytschko T., Chang T.P.: Continuum theory for strain softening, Journal of Engineering Mechanics-ASCE, 110(12), 1984, 1666-1692

[6] Bazant Z.P. and Pijaudier Cabot G.: Nonlocal Continuum Damage, Localization Instabilities and Convergence, Journal of Engineering Mechanics, 1988, 55, 287-293

[7] Z. P. Bazant and P. Prat: Microplane model for brittle plastic materials. I: Theory, II: Verification. Journal of Engineering Mechanics ASCE, 1988, 114, 1672-1702.

[8] Bazant Z.P., Jirasek M.: Nonlocal Integral Formulations of Plasticity and Damage: Survey of Progress, Journal of Engineering Mechanics, 2002, 128 (11),

[9] Bazant Z.P., Oh B.H.: Crack band theory for fracture of concrete, Materials and Structures, January-February, $1983,155-177$

[10] Belytschko T., Guo Y., Liu W.K., Xiao S.P.: A unified stability analysis of meshless particle methods, Int. J. Numer. Meth. Engng 2000, 48, 1359-1400

[11] Belytschko T., Organ D., Krongauz Y.: A coupled finite element-element-free Galerkin method, Computational Mechanics 17 (1995), 186-195

[12] Belytschko T. Krongauz Y., Dolbow J., Gerlach C.: On the completeness of Meshfree Particle methods, International Journal for numerical methods in Engineering 43 (1998), 785-819

[13] Belytschko T., Lu Y.Y., et al.: Element-free Galerkin methods, International Journal for Numerical Methods in Engineering, 37 (1994), 229-256

[14] Belytschko T.: Crack propagation by element free Galerkin methods, Engineering Fracture mechanics, 51/2 (1995), 295-315

[15] Belytschko T., Lu Y.Y.: Element-free Galerkin methods for static and dynamic fracture, Int. J. Solids Strucutres 32 (1995), 2547-2570

[16] Belytschko T., Liu W.K., Moran B.: Nonlinear Finite Elements for Continua and Structures, 2000, John Wiley and Sons Ltd., New York, USA

[17] Belytschko T., Xiao S.P.: Stability analysis of Particle Methods with Corrected Derivatives, Computers and Mathematics with Applications 43 (2000), 329-350

[18] Belytschko T., Black T.: Elastic crack growth in finite elements with minimal remeshing, International Journal for Numerical Methods in Engineering, 1999, Volume 45(5), 601-620

[19] Belytschko T., Moes N., Usui S., Parimi C.: Arbitrary discontinuities in finite elements, International Journal for Numerical Methods in Engineering, 2001, Volume 50(4), 993-1013

[20] Bittencourt T.N., Wawrzynek P.A., Ingraffea A.R.: Quasi-automatic simulation of crack propagation for 2D LEFM problems, Engineering Fracture Mechanics, 1996, 55(2), 321-334

[21] Bosco C., Debernardi P.G.: Experimental Investigations on the Ultimate Rotational Capacity of R.C. Beams, Dipartimento di Ingegneria Strutturale, Politecnico de Turin, 1992 
[22] Brown K., Attaway S., Plimpton S., Hendrickson B.: Parallel strategies for crash and impact simulations, Comp. Methods Appl. Mech. Engrg. 184 (2000), 375-390

[23] Carter B.J., Wawrzynek P.A., Ingraffea A.R.: Automated 3-D crack growth simulation, International Journal for numerical methods in Engineering, 2000, 47(1-3), 229-253

[24] Chen J.S., Pan C., Wu C.T., Liu W.K.: Repdroducing kernel particle methods for large deformation analysis of nonlinear structures, Computer Methods in Applied Mechanics and Engineering, Vol. 139, 195-227, 1996

[25] Chen J.S., Pan C., Roque, C.M.O.L., Wang H.P.: A Lagrangian Reproducing kernel particle method for metal forming analysis, Computational Mechanics, Vol. 22, 289-307, 1998

[26] Chen W.F., 1994. Constitutive Equations for Engineering Materials, Volume 2: Plasticity and Modeling, Elsevier, Amsterdam-London-New York-Tokio

[27] Cox J.V., Herrmann L.R.: Development of a plasticity bond model for steel reinforcement, Mechanics of Cohesive-Frictional Materials, 1998, 3, 155-180

[28] Cox J.V., Herrmann L.R.: Validation of a plasticity bond model for steel reinforcement, Mechanics of CohesiveFrictional Materials, 1999, 4, 361-389

[29] Den Ujil J., Bigaj A.J., 1996. A bondmodel for ribbed bars based on concrete loaded in compression, Heron, Vol. 41, No.3

[30] Eibl J., Stempniewski L., Rabczuk T.: Der Endbereich von im Werk vorgespannten FertigteiltraegernHohlplatten, Abschlussbericht, Institut fuer Massivbau und Baustofftechnologie, Universitaet Karlsruhe, 2001

[31] Eligehausen R., Mayer U.: Parameterstudie zur Mitwirkung des Betons zwischen den Rissen unter Kurzzeitbelastung insbesondere in Abhaengigkeit von der Duktilitaet des Betonstahles, Forschungsbericht, Universitaet Stuttgart, 1997

[32] Gravouil A., Moes N., Belytschko T.: Non-planar 3D crack growth by the extended finite element and level sets - Part II: Level set update, International Journal for Numerical Methods in Engineering, 2002, 53, 2569-2586

[33] De Groot A.K., Kusters G.M.A. Monnier T.: Numerical Modeling of Bond-Slip behavior, Heron, 26-1b, I.B.B.C., 1981, Institute Delft, Netherlands, 90 pp.

[34] Hegen D.: Element free Galerkin methods in combination with finite element approaches, Computer Methods in applied Mechanics and Engineering, 1996, 135, 143-166

[35] Hillerborg A., Modeer A., Peterson P.E.: Analysis of crack formation and crack growth in concrete by means of fracture mechanics and finite elements, Cement and Concrete Research, 1976 (6), 773-782

[36] Idda, K.: Verbundverhalten von Betonrippenstaehlen bei Querzug, University of Karlsruhe, Institut fuer Massivbau und Baustofftechnologie, PhD-thesis, 1999

[37] Ingraffea A.R., Gerstle W.H., Gergely P., Saoma V.: Fracture Mechanics of Bond in Reinforced Concrete, Journal of Structural Engineering, ASCE, 1984, 111 (5), 871-890

[38] Jirasek M., Zimmermann T.: Rotating Crack Model with Transition to Scalar Damage, ASCE, Journal of Engineering Mechanics, 1998, 124 (3), 277-284

[39] Jirasek M.: Modeling of fracture and damage in quasibrittle materials, 1993, PhD thesis, Northwestern University, USA

[40] Jirasek M.: Comparative study on finite elements with embedded discontinuities, Computer Methods in Applied Mechanics and Engineering, 2000, 188, 307-330

[41] Johansson M.: New Reinforcement Detailing in Frame Corners in Civil Defence Shelters-Experiments and Fracture Mechanics Analyses, Chalmers University of Technology, Division of Concrete Structures, Report 95:2, Goeteborg, 1995

[42] Johnson G.R.: Linking of Lagrangian Particle Methods to Standard Finite Element Methods for High Velocity Impact Copmutations, Nuclear Engineering and Design 150, 1994, Post-SMIRT Impact IV Seminar Berlin 
[43] Johnson G.R., Stryk R.A., Beissel S.R.: SPH for high velocity impact computations, Computer Methods in Applied Mechanics and Engineering, 1996, 139, 347-374

[44] Karutz H.: Adaptive Kopplung der Elementfreien Galerkin Methode mit der Methode der Finiten Elemente bei Rissfortschrittsproblemen, Dissertation, 2000, Institut fuer Statik und Dynamik der Ruhr Universitaet Bochum, VDI-Verlag, Reihe 18, Band 255

[45] Keuser M.: Verbundelemente fuer nichtlineare Finite-Element-Berechnungen von Stahlbetonkonstruktionen, VDI Verlag, Duesseldorf, 1985

[46] Krysl P., Belytschko T.: The Element Free Galerkin Method for dynamic propagation of arbitrary 3-D cracks, International Journal for Numerical Methods in Engineering, 1999, 44 (6), 767-800

[47] Lemaitre J.: Evaluation of dissipation and damage in metal submitted to dynamic loading, Proceedings ICM 1,1971

[48] Malvar L.J.: Bond of Reinforcement under Controlled Confinement, ACI Materials Journal, 1992, 89 (6), 593-601

[49] Oliver J.: Modelling strong discontinuities in solid mechanics via strain softening constituitive equations, part 1: fundamentals. part 2: numerical simulation, International Journal for Numerical Methods in Engineering, $1996,39,3575-3624$

[50] Potyondy D.O., Wawrzynek P.A., Ingraffea A.R.: An Algorithm to generate quadrilaterial or triangular element surface meshes in arbitrayr domains with applications to crack-propagation, International Journal for Numerical Methods in Engineering, 1995, 38(16), 2677-2701

[51] Simkins D.C , Li S., Lu H., Liu W.K.: Reproducing kernel element method. Part IV: Globally compatible Cn (n1) triangular hierarchy, Computer Methods in Applied Mechanics and Engineering, Volume 193, Issues 12-14, 26 March 2004, 1013-1034

[52] Lucy: A numerical Approach to the Testing of Fission Hypothesis, Astronomical Journal, 82, 1977, 1013-1024

[53] Rabczuk T., Eibl J.: Simulation of high velocity concrete fragmentation using SPH/MLSPH, Int. J. Numer. Meth. Engng, 2003, 56, 1421-1444

[54] Rabczuk T., Belytschko T., Xiao S.P.: Stable particle methods based on Lagrangian kernelss, accepted in Computer Methods in Applied Mechanics and Engineering

[55] Rabczuk T., Belytschko T.: Cracking particles: a simplified meshfree methods for arbitrary evolving cracks, Int. J. Numer. Meth. Engng., 2004, 61(13), 2316-2343

[56] Schaefer K., Baumann P.: Ausbreitung von Druckkraeften in Betonscheiben- Vergleichende Versuche mit Lasteinleitungen ueber Lastplatten, Bewehrungsstabumlenkungen und Bewehrungsknoten, Forschungsbericht DFG, Institut fuer Massivbau, Universitaet Stuttgart, Germany, 1986

[57] Stucki D., Thuerlimann B.: Versuche an Eckverbindungen aus Stahlbeton, Institut fuer Baustatik und Konstruktion, ETH Zuerich, 1990

[58] G. Ventura, J. Xu, T. Belytschko: A vector level set method and new discontinuity approximations for crack growth by EFG, Int. J. Numer. Meth. Engng., 2002, 54 (6), 923-944

[59] Xiao S.P., Belytschko T.: Material Stability Analysis of Particle Methods, submitted

[60] Xu X.P., Needleman A.: Numerical simulations of dynamic crack growth along an interface, INT J FRACTURE, 199674 (4): 289-324 